\title{
Node Density and Delay in Large-Scale Wireless Networks with Unreliable Links
}

\author{
Shizhen Zhao, Xinbing Wang \\ Department of Electronic Engineering \\ Shanghai Jiao Tong University, China \\ Email: \{shizhenzhao,xwang8\}@sjtu.edu.cn
}

\begin{abstract}
We study the delay performance in large-scale wireless multi-hop networks with unreliable links from percolation perspective $^{1}$. Previous works $[12][3][11]$ have showed that the end-to-end delay scales linearly with the source-to-destination distance, and thus the delay performance can be characterized by the delay-distance ratio $\gamma$. However, the range of $\gamma$, which may be the most important parameter for delay, remains unknown. We expect that $\gamma$ may depend heavily on the node density $\lambda$ of a wireless multi-hop network. In this paper, we investigate the fundamental relationship between $\gamma$ and $\lambda$. Obtaining the exact value of $\gamma(\lambda)$ is extremely hard, mainly because of the dynamically changing network topologies caused by the link unreliability. Instead, we provide both upper bound and lower bound to the delay-distance ratio $\gamma(\lambda)$. Simulations are conducted to verify our theoretical analysis.
\end{abstract}

Index Terms-Connectivity, Delay, Density

\section{INTRODUCTION}

Large-scale wireless multi-hop networks are fast becoming the most preferred way for communication in outdoor environment [1]. Each user in a wireless multi-hop network could initiate a communication in a multi-hop fashion without of the aid of any network infrastructure. Thus, the maintenance cost for communication networks can be significantly reduced. However, relays of a wireless multi-hop network may be also users, which are highly unreliable due to the unexpected user behavior and the time-varying wireless channel. Thus, it is highly possible that a connection may get lost during data transmission, in which case the data has to wait at some node and resume its transmission after the reestablishment of the network connection. The lost of connection could cause significant delay to data transmission. Therefore, it is very critical to provide some delay guarantee for wireless multi-hop networks. Note that the data communication reliability can be improved by some back-up routes, and it is usually easier to find more routes when the node density is higher. Hence, we expect that the data communication delay may depend heavily on the node density. Our goal in this paper is to quantify the fundamental relationship between node density and delay.

To make our study meaningful, we assume that the largescale wireless network is connected. Full connectivity [4] can ensure the successful communication between each node pair in a wireless network. However, it is overly power consuming to achieve full connectivity in large-scale networks (i.e., the

\footnotetext{
${ }^{1}$ An earlier version of this paper appeared in ACM MobiCom 2011 [19]
}

power required to maintain full connectivity increases with the size of the network). Hence, it is necessary to introduce a slightly weaker connectivity criterion, in which we only ensure that a large portion of the network nodes are connected via multiple hops with each other. Thanks to percolation theory [10][15], it is possible to achieve this weaker connectivity in large-scale networks with power bounded per node.

Percolation theory [10], especially continuum percolation, is a powerful mathematical tool when analyzing the connectivity and the delay of wireless networks. An important and general model of Continuum Percolation is Random Connection Model (RCM). RCM describes the behavior of connected components in an infinitely large random geometric graph, in which nodes are distributed according to poisson point process with node density $\lambda$, and two nodes share a link with probability $h(r)$ ( $r$ is the distance between the two nodes). A classical result of RCM points out a fundamental phase transition effect at a critical node density $\lambda_{c} \in(0, \infty)$. For $\lambda>\lambda_{c}$ (supercritical), there exists a unique connected component containing a large portion of nodes in the infinitely large network (we also say the network is percolated). However, for $\lambda<\lambda_{c}$ (subcritical), all connected component in the network are finite almost surely.

Applying percolation theory to large-scale wireless multihop networks, we introduce two important concepts, i.e., the instantaneous connectivity and the long-term connectivity. The Instantaneous connectivity requires wireless networks percolated at all the time instances. While the long-term connectivity only requires wireless network percolated in the long run (we will elaborate it more clearly later in sectionII-B1). The instantaneous critical density, denoted by $\lambda_{I}$, is the critical density for the instantaneous connectivity and the long-term critical density, denoted by $\lambda_{L}$, is the critical density for the long-term connectivity. Long-term connectivity is a weaker criterion for connectivity, thus $\lambda_{L}<\lambda_{I}$. To ensure connectivity, we assume that $\lambda>\lambda_{L}$ throughout this paper.

In large-scale wireless networks, delay is mainly composed of the waiting delay and the propagation delay. The waiting delay is caused by the loss of connection at some time instances, which is due to the lack of instantaneous connectivity. Packets must wait for some time until the connection is reestablished. Usually, such a waiting time is in the order of seconds, minutes or more. The propagation delay is the time required for a packet to transverse a link whenever the link is active. Mostly, the propagation delay is in the order of milliseconds. 
Therefore, it is negligibly small compared to the waiting delay. For ease of analysis, we first ignore the impact of propagation delay and will consider its effect in the last.

We study the delay performance for flooding in large-scale wireless networks. Ignoring the propagation delay, previous works [12][3][11] have showed that if $\lambda_{L}<\lambda<\lambda_{I}$, the end-to-end delay scales linearly with the source-to-destination distance $(\gamma(\lambda)>0)$, and that if $\lambda>\lambda_{I}$, the end-to-end delay scales sub-linearly with distance $(\gamma(\lambda)=0)$. However, these results are far from enough to provide reasonable delay guarantee. It remains extremely important to find out the exact value or the lower and upper bounds of $\gamma(\lambda)$.

In this paper, we present a theoretical analysis about the flooding delay in wireless multi-hop networks with unreliable links. We first ignore the propagation delay and find the upper and lower bounds of $\gamma(\lambda)$. For the upper bound, we first find a path between two nodes using percolation theory. And then we calculate the number of hops along this path using another coupling technique and the delay at each hop. We obtain the result of upper bound by multiplying the above two items. For the lower bound, we first introduce a concept called cluster to cluster transmission process and establish the relationship between delay and the cluster to cluster transmission process, which reveals the essence of the flooding delay in wireless multi-hop networks. Then, based on the delay of the cluster to cluster transmission, we obtain a lower bound of $\gamma(\lambda)$.

We then generalize the previous result to the case with nonzero propagation delay. Propagation delay will aggravate the delay performance in Large-Scale wireless Networks, making $\gamma(\lambda)>0$ even when $\lambda>\lambda_{I}$. Using similar methods, we present new upper and lower bounds to $\gamma(\lambda)$ for all $\lambda>\lambda_{L}$.

Finally, we conduct simulation to verify our theoretical results.

The original contributions that we have made in the paper are highlighted as follows:

- We present two properties to $\gamma(\lambda)$, i.e., $\gamma(\lambda)=0$ whenever propagation delay is 0 and $\lambda>\lambda_{I} ; \gamma(\lambda)$ is a monotone decreasing function.

- Ignoring propagation delay, we provide the upper bound and the lower bound to reflect the range of variation on $\gamma(\lambda)$.

- Taking propagation delay into consideration, we obtain further results.

- We conduct simulations to obtain experimental values of $\gamma(\lambda)$ in the above two cases. The new observation arises from our comparison between theoretical and simulation results is that the delay-distance ratio $\gamma(\lambda)$ can be approximated by the lower bound in relative dense networks while the experimental values of $\gamma(\lambda)$ get closer to the upper bound in relative sparse networks. This also justifies the soundness of our theoretical conclusion.

The rest of the paper is organized as follows. In section II, we introduce our network model, several useful mathematical tools and some important notations. In section III, we first give two properties of $\gamma(\lambda)$, and then present our main results concerning the upper and lower bound of $\gamma(\lambda)$. The analysis for obtaining the upper and lower bounds is given in section IV. Simulation results are presented in Section VI to justify our theoretical findings. We summarize the paper in Section VII. Some proofs of the theorems and lemmas are presented in section $\mathrm{V}$.

\section{BACKGRound AND Network Model}

In this section, we introduce some background knowledge and the network model.

\section{A. Background}

1) Poisson Point Process: In large-scale wireless multi-hop networks, it is extremely difficult to predict the exact locations of different users (nodes) due to the random behavior of different users. To model the location-randomness, we use Poisson Point Process [18]. One way to visualize the Poisson Point Process is to assume that all users are distributed uniformly in a given area, e.g., $\lambda n^{2}$ nodes are evenly distributed in a $n \times n$ area. If we let $n \rightarrow \infty$, then the distribution of these random processes (indexed by $n$ ) will converge in distribution to Poisson Point Process with rate $\lambda$.

Poisson Point Process has the following two properties:

- The superposition of two independent Poisson Point Processes is still a Poisson Point Process.

- The number of nodes counted in disjoint areas are independent from each other (spatial independence).

As readers will see, the above two properties are extremely useful in our analysis.

2) Random Connection Model: Before introducing the network model, we need a brief introduction to Continuum Percolation. Connectivity is a significant issue in wireless network, which has been extensively explored by [4][5][6][7][8][9]. In this paper, we present the definition of connectivity in the percolation perspective. To make the results in this paper applicable to the most scenarios, we focus on a fairly general model in Continuum Percolation Theory, i.e., the Random Connection Model(RCM) [15].

In the RCM, nodes are distributed according to Poisson point process in $\mathbb{R}^{d}$. Here we only focus on the case of $\mathbb{R}^{2}$ with node density $\lambda>0$. Each node $x$ connects to another node $y$ according to some predefined connection function $h(r)$, where $r=\|x-y\|$ is the distance between $x$ and $y$. Specifically, nodes $x$ and $y$ are connected with probability $h(r)$. We assume that $h(r)$ satisfies $0<\int_{\mathbb{R}^{2}} h(r) d x d y<\infty$, which ensures the phase transition effect [15] in the Random Connection Model.

We denote a RCM by $\mathcal{G}\left(\lambda, r_{0}, h(r)\right)^{2}$, where $\lambda$ is the node density, $r_{0}=\sup \{r \mid h(r)>0\}, \mathrm{h}(\mathrm{r})$ is the connection function. Then $\mathcal{G}\left(\lambda, r_{0}, h(r)\right)$ is a set of nodes connected by random links. For convenience, we assume that the origin $0 \in \mathcal{G}\left(\lambda, r_{0}, h(r)\right)$.

Obviously, $\mathcal{G}\left(\lambda, r_{0}, h(r)\right)$ is composed of a set of disjointed connected components. Let us denote $W(A), A \subseteq$ $\mathcal{G}\left(\lambda, r_{0}, h(r)\right)$, the set of nodes attainable from nodes in set A, i.e.,

$$
W(A)=\left\{x \in \mathcal{G}\left(\lambda, r_{0}, h(r)\right) \mid \exists a \in A, a \leftrightarrow x\right\},
$$

\footnotetext{
${ }^{2}$ In general, a RCM can be fully determined by $\lambda$ and $h(r)$ only. We just use $r_{0}$ to emphasize that we only focus on functions $h(r)$ that are 0 for $r$ larger than some finite value $r_{0}$.
} 
where, $a \leftrightarrow x$ means that nodes $a$ and $x$ are in the same connected component.

Besides, we use $|W|$ to represent the cardinality of the set $W$. Further, we define $\theta_{h}(\lambda)=\mathbb{P}_{\lambda, h}(|W(\{0\})|=\infty)^{3}$ and $\chi_{h}(\lambda)=E_{\lambda, h}(|W(\{0\})|)^{4}$.

Then, the critical density can be determined in two ways, i.e.,

$$
\begin{gathered}
\lambda_{\theta}(h)=\inf \left\{\lambda \mid \theta_{h}(\lambda)>0\right\} \\
\lambda_{\chi}(h)=\inf \left\{\lambda \mid \chi_{h}(\lambda)=\infty\right\} .
\end{gathered}
$$

According to Theorem 6.2 in [15], $0<\lambda_{\theta}(h)=\lambda_{\chi}(h)=$ $\lambda_{c}(h)<\infty$. Furthermore, there exists a unique infinite connected component containing a large portion of the nodes in $\mathcal{G}\left(\lambda, r_{0}, h(r)\right)$ with probability 1 if $\lambda>\lambda_{c}(h)$ (supercritical). This infinite connected component is also called the giant component, denoted by $\mathcal{C}\left(\mathcal{G}\left(\lambda, r_{0}, h(r)\right)\right)$. In this case, we call $\mathcal{G}\left(\lambda, r_{0}, h(r)\right)$ percolated. On the other hand, if $\lambda<\lambda_{c}(h)$ (subcritical), all the connected components are finite almost surely.

Another useful model in Continuum Percolation is Poisson Boolean Model, denoted by $\mathcal{B}(\lambda, r)$. In the Poisson Boolean Model $\mathcal{B}(\lambda, r)$, nodes are distributed according to Poisson Point Process with density $\lambda$, and two nodes can communicate if and only if their distance is smaller than $r$. Poisson Boolean Model can be viewed as a special case of Random Connection Model. Thus, the conclusions for Random Connection Model also hold for Poisson Boolean Model.

\section{B. Model}

1) Network Model: We model the large-scale wireless multi-hop network as a random graph with dynamically varying edges. We assume that the network nodes are distributed according to Poisson Point Process with node density $\lambda$ in an infinite two-dimensional space $\mathbb{R}^{2}$. For each node $u$, we use $u$ to represent both this node and its location without causing confusion. We say two nodes share a link if and only if their distance is smaller than $r_{0}$. However, due to the unreliability of the wireless channel and the unexpected user behaviors, each link suffers the possibility to fail. We say a link open at a time slot if this link can successfully transmit a packet, and closed otherwise. We model the link failure as each link opening or closing intermittently. Specifically, we assume that time is slotted, and the duration of each time slot is 1 . Consider a link with length $r$, at time slot $t$. We let it open with probability $g(r)$ (Fig. 1), independent of its former states. In reality, the farther two nodes are apart, the more difficult for a successful communication. Moveover, when $r>r_{0}$, there exists no link. Thus, it is reasonable to assume that $g(r)$ is a monotone decreasing function and $g(r)=0$ whenever $r>r_{0}$. Further, we place another constraint on $g(r)$, i.e.,

$$
1>g(0) \geq g(r) \geq g\left(r_{0}\right)>0,0 \leq r \leq r_{0} .
$$

As readers will see, constraint (3) is used to ensure that the expected traversing delay over each possible link is bounded above.

\footnotetext{
${ }^{3} \mathbb{P}$ is the probability of a event.
}

${ }^{4} E(x)$ is the expectation of random variable $x$

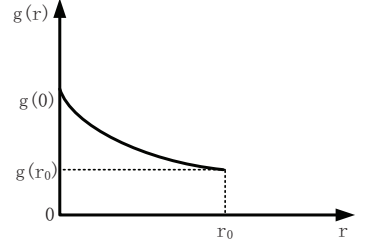

(a) Illustration of connection function $g(r)$.

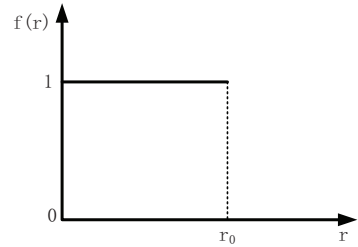
function $f(r)$. (b) Illustration of connection

Fig. 1. Illustration of two connection functions.

Based on the above discussion, the network at each time slot $t$ can be represented by a $\operatorname{RCM} \mathcal{G}_{t}\left(\lambda, r_{0}, g(r)\right)$. Here, we use subscript $t$ to indicate that the network is dynamic. Note that if $\lambda>\lambda_{c}(g(r)), \mathcal{G}_{t}\left(\lambda, r_{0}, g(r)\right)$ is percolated for all $t$ (we also say the network has instantaneous connectivity); while if $\lambda<\lambda_{c}(g(r)), \mathcal{G}_{t}\left(\lambda, r_{0}, g(r)\right)$ is not percolated for all $t$. Thus, the instantaneous critical density $\lambda_{I}=\lambda_{c}(g(r))$.

Next, we introduce the concept of the long-term connectivity. We first construct a new graph. The location of all nodes in this graph is the same as that in $\mathcal{G}_{t}\left(\lambda, r_{0}, g(r)\right)$. Two nodes $x$ and $y$ share a link in this graph if and only if there exist $t$, such that $x$ and $y$ share an open link in $\mathcal{G}_{t}\left(\lambda, r_{0}, g(r)\right)$. Note that $x$ and $y$ has the potential to share a link in $\mathcal{G}_{t}\left(\lambda, r_{0}, g(r)\right)$ for some $t$, whenever $g(r)>0$ (equivalently, $r<r_{0}$ ). Thus this new geometric graph can be represented by a RCM $\mathcal{G}\left(\lambda, r_{0}, f(r)\right.$ ) (it can be also represented by Poisson Boolean Model $\mathcal{B}\left(\lambda, r_{0}\right)$ ). Here, $f(r)=1$ when $r<r_{0}$, and $f(r)=0$ when $r>r_{0}$ (Fig. 1). We say the wireless network has longterm connectivity if and only if $\mathcal{G}\left(\lambda, r_{0}, f(r)\right)$ is percolated, and the critical density $\lambda_{L}=\lambda_{c}(f(r))$ is defined as the longterm critical density.

Note that the long-term connectivity graph $\mathcal{G}\left(\lambda, r_{0}, f(r)\right)$ is a super-graph of all the instantaneous connectivity graph $\mathcal{G}_{t}\left(\lambda, r_{0}, g(r)\right)$. Thus, whenever $\mathcal{G}_{t}\left(\lambda, r_{0}, g(r)\right)$ is percolated, $\mathcal{G}\left(\lambda, r_{0}, f(r)\right)$ is percolated. Based on this observation, it is easy to know that $\lambda_{L} \leq \lambda_{I}$. Since the prerequisite for communication in large-scale wireless network is connectivity, it is enough to only focus on the case $\lambda>\lambda_{L}$.

2) Modeling Delay in Large-Scale Wireless Networks: The definition of delay of large-scale network is based on the First Passage Percolation [2]. Specifically, given a Random Connection Model $\mathcal{G}\left(\lambda, r_{0}, h(r)\right)$, we attach each link $e$ of $\mathcal{G}\left(\lambda, r_{0}, h(r)\right)$ a random variable $T_{c}(e)$, representing the time needed to pass through the link $e$. Consider a path $\pi$, the passage time is defined as

$$
T_{p}(\pi)=\sum_{e \in \pi}\left(T_{c}(e)\right) .
$$

We then define the first-passage time $T_{\lambda}(x, y)$ for any two nodes $x$ and $y(x, y$ are not necessarily adjacent) as the minimum delay among all possible routes, i.e.,

$$
T_{\lambda}(x, y)=\inf \left\{T_{p}(\pi): \pi \text { is a path from } x \text { to } y\right\} \text {. }
$$

We now give the detailed definition of $T_{c}(e)$. Generally, the time needed to cross the link $e$ is composed of two parts. The first part is called waiting delay, which is caused by the unreliability of this link. The second part is called propagation 
delay ${ }^{5}$, which is the time required for a packet to transverse the link $e$ whenever the link is on. We assume that the duration of a time slot is long enough for a packet to be successfully transmitted over a link. Equivalently, the propagation delay over a link is smaller than 1 time slot. For ease of analysis, we assume that all active links can transmit simultaneously with the same propagation delay $\tau$, where $\tau<1$ time slot. Based on the above discussion, we can express the crossing time $T_{c}(e)$ as a geometrically distributed random variable. Specifically, assume that the length of the link $e$ is $0<r<r_{0}$, then we have

$$
\mathbb{P}\left(T_{c}(e)=k+\tau\right)=(1-g(r))^{k} g(r) .
$$

Now we are ready to introduce the delay-distance ratio $\gamma(\lambda)$. Consider two nodes $x, y \in \mathcal{C}\left(\mathcal{G}\left(\lambda, r_{0}, f(r)\right)\right)^{6}$. Previous works [12][3][11] have proved that, the two limits $\lim _{d(x, y) \rightarrow \infty} \frac{T_{\lambda}(x, y)}{d(x, y)}$ and $\lim _{d(x, y) \rightarrow \infty} \frac{E\left(T_{\lambda}(x, y)\right)}{d(x, y)}$ exist almost surely. Furthermore,

$$
\lim _{d(x, y) \rightarrow \infty} \frac{T_{\lambda}(x, y)}{d(x, y)}=\lim _{d(x, y) \rightarrow \infty} \frac{E\left(T_{\lambda}(x, y)\right)}{d(x, y)}, \text { a.s. }
$$

We denote the above limit by $\gamma(\lambda) \cdot \gamma(\lambda)$ characterizes the first passage delay $T_{\lambda}(x, y)$ with respect to the distance $d(x, y)$. We need to emphasize that $\gamma(\lambda)$ depends on $\lambda$. In fact, previous results also show that if $\tau=0$ and $\lambda>\lambda_{I}, \gamma=0$. Otherwise, $\gamma>0$. However, none of the previous works studies the exact relationship between $\gamma(\lambda)$ and $\lambda$, especially, how $\gamma(\lambda)$ varies with respect to $\lambda$ in the region $\left(\lambda_{L}, \lambda_{I}\right]$. In this paper, we will give a more detailed quantification of $\gamma(\lambda)$.

\section{Useful Notations}

Some useful notations are listed as follows.

- (Section II-A2) $\mathcal{G}\left(\lambda, r_{0}, h(r)\right)$ is a Random Connection Model, and $h(r)$ is the connection function; $\mathcal{B}(\lambda, r)$ is the Poisson Boolean Model; we use $\mathcal{C}\left(\mathcal{G}\left(\lambda, r_{0}, h(r)\right)\right)$ and $\mathcal{C}(\mathcal{B}(\lambda, r))$ to represent the giant component of $\mathcal{G}\left(\lambda, r_{0}, h(r)\right)$ and $\mathcal{B}(\lambda, r)$ respectively.

- (Section II-B1) $\mathcal{G}_{t}\left(\lambda, r_{0}, g(r)\right)$ is the instantaneous geometric graph at time slot $t$ and its critical density is $\lambda_{I}$; $\mathcal{G}\left(\lambda, r_{0}, f(r)\right)$ is the long-term geometric graph and its critical density is $\lambda_{L}$.

- $\mathbb{P}(\bullet)$ represents the probability of some event; $E(\bullet)$ represents the expectation of a random variable; $z_{x}\left(z_{y}\right)$ represents the $x(y)$-coordinate of $z ; d(u, v)=\|u-v\|$ is the Euclidean distance between node $u$ and $v$.

- (Section IV-B) $H\left(z_{0}, a\right)$ is a circular region defined as $H\left(z_{0}, a\right)=\left\{z=\left(z_{x}, z_{y}\right) \in \mathbb{R}^{2} \mid\left\|z-z_{0}\right\|<a\right\}$. The random variable $S_{g, t, u}(\lambda)$ is defined as $S_{g, t, u}(\lambda)=$ $\sup \left\{a \mid \exists\right.$ node $v \in H^{c}(u, a), v$ and $u$ are connected in $\left.\mathcal{G}_{t}\left(\lambda, r_{0}, g(r)\right)\right\}$. In fact, all $S_{g, t, u}(\lambda)$ 's (indexed by $t$ and $u$ ) have the same distribution. Thus, we write the expectation of $S_{g, t, u}(\lambda)$ as $E\left(S_{g}(\lambda)\right)$ for short.

\footnotetext{
${ }^{5}$ To be precise, this propagation delay includes both the processing delay at the sending node, and the propagation delay along the link

${ }^{6}$ Such a giant component exists with probability 1 , because we focus on the case $\lambda>\lambda_{L}$ in this paper.
}

- (Section II-B2) $T_{c}(e)$ is the passage time for a link $e$; $T_{p}(\pi)$ is the passage time for a path $\pi ; T_{\lambda}(x, y)$ is the first passage time from node $x$ to $y$; (Section IV-B1) $T_{p}(\Pi)$ is the passage time for a cluster to cluster transmission process $\Pi$;

- (Section IV-A) $N_{\lambda}(d(u, v))$ is the minimum number of hops from node $u$ to $v$.

- $\pi$ represents a path; $\Pi$ represents a cluster to cluster transmission process.

\section{MAIN RESULTS}

In this section, we first give two properties on the delaydistance ratio $\gamma(\lambda)$. After that, we present our main results concerning the relationship between node density $\lambda$ and $\gamma(\lambda)$, in which an upper bound and a lower bound for $\gamma(\lambda)$, are given.

\section{A. Properties of $\gamma(\lambda)$}

$\gamma(\lambda)$ can be viewed as a function mapping from $\left(\lambda_{L}, \infty\right)$ to $\mathbb{R}$. The properties of $\gamma(\lambda)$ are listed below.

Theorem 1. $\gamma(\lambda)$ has the following two properties:

1) if $\tau=0$, then $\forall \lambda>\lambda_{I}, \gamma(\lambda)=0$;

2) $\gamma(\lambda)$ is a monotone decreasing function.

Proof: The first property has already been proved by previous literatures [12][11][3][17]. Thus, we only present the proof of the property (2) here.

Given $\lambda_{1}>\lambda_{2}$, consider two Random Connection Models $\mathcal{G}_{t}\left(\lambda_{1}, r_{0}, g(r)\right)$ and $\mathcal{G}_{t}\left(\lambda_{2}, r_{0}, g(r)\right)$. We use coupling technique to prove $\gamma\left(\lambda_{1}\right) \leq \gamma\left(\lambda_{2}\right)$. Nodes in $\mathcal{G}_{t}\left(\lambda_{1}, r_{0}, g(r)\right)$ and $\mathcal{G}_{t}\left(\lambda_{2}, r_{0}, g(r)\right)$ are distributed according to Poisson Point Processes, denoted by $\Gamma_{\lambda_{1}}$ and $\Gamma_{\lambda_{2}}$, with node densities $\lambda_{1}$ and $\lambda_{2}$, respectively. Note that $\Gamma_{\lambda_{1}}$ can be viewed as the superposition of $\Gamma_{\lambda_{2}}$ and another Poisson Point Process $\Gamma_{\lambda_{1}-\lambda_{2}}$ with node density $\lambda_{1}-\lambda_{2}$.

Consider nodes $x, y \in \Gamma_{\lambda_{2}}$, since $\Gamma_{\lambda_{2}} \subseteq \Gamma_{\lambda_{1}}$, we obtain $x, y \in \Gamma_{\lambda_{1}}$. For any path $\pi$ connecting $x$ and $y$ in $\mathcal{G}_{t}\left(\lambda_{2}, r_{0}, g(r)\right)$, this path also exists in $\mathcal{G}_{t}\left(\lambda_{1}, r_{0}, g(r)\right)$. By the definition of $T_{\lambda}(x, y)$, we must have

$$
T_{\lambda_{1}}(x, y) \leq T_{\lambda_{2}}(x, y)
$$

Divide the above inequality by $d(x, y)$, and let $d(x, y) \rightarrow$ $\infty$, we obtain

$$
\gamma\left(\lambda_{1}\right) \leq \gamma\left(\lambda_{2}\right)
$$

\section{B. Main results on $\gamma(\lambda)$}

We have obtained several properties of $\gamma(\lambda)$. Now we are ready to present our main results. 
Theorem 2. Given a $R C M \mathcal{G}_{t}\left(\lambda, r_{0}, g(r)\right)$ with $\lambda_{L}(1+\epsilon) \leq$ $\lambda<\lambda_{I}(\epsilon>0)$ and $\tau=0$, the corresponding $\gamma(\lambda)$ satisfies

$$
\begin{aligned}
& \frac{1}{E\left(S_{g}(\lambda)+r_{0}\right)} \leq \gamma(\lambda) \\
\leq & \inf _{\lambda^{\prime} \in\left[\lambda_{L}(1+\epsilon), \lambda\right]} \kappa_{\epsilon} \sqrt{\frac{\lambda^{\prime}}{\lambda_{L}}}\left(\frac{1}{g\left(r_{0} \sqrt{\frac{\lambda_{L}(1+\epsilon)}{\lambda^{\prime}}}\right)}-1\right) \text {, }
\end{aligned}
$$

where $\kappa_{\epsilon}$ is a constant independent of $\lambda$.

Theorem 2 ignores the propagation delay. If we take propagation delay into consideration, we have the following results.

Theorem 3. Given a $R C M \mathcal{G}_{t}\left(\lambda, r_{0}, g(r)\right)$ with $\lambda \geq \lambda_{L}(1+\epsilon)$ $(\epsilon>0)$ and $0<\tau<1$. Then the corresponding $\gamma(\lambda)$ satisfies

$$
\begin{aligned}
& \frac{1}{E\left(\min \left\{S_{g}(\lambda), \frac{r_{0}}{\tau}\right\}\right)+r_{0}} \leq \gamma(\lambda) \\
& \leq \inf _{\lambda^{\prime} \in\left[\lambda_{L}(1+\epsilon), \lambda\right]} \frac{\kappa_{\epsilon} \sqrt{\lambda^{\prime} / \lambda_{L}}}{g\left(r_{0} \sqrt{\frac{\lambda_{L}(1+\epsilon)}{\lambda^{\prime}}}\right)}
\end{aligned}
$$

where $\kappa_{\epsilon}$ is a constant independent of $\lambda$.

We need to emphasize that the two $\kappa_{\epsilon}$ 's in Theorem 2 and Theorem 3 are the same. The rigorous definition of $\kappa_{\epsilon}$ is postponed to Section IV-A (see Lemma 3).

Our results provide a way to estimate delay in Large-scale wireless multi-hop networks. Our result is also very general. By changing the connection function $g(r)$, our results can be easily applied to different scenarios in real networks.

\section{UPPER AND LOWER BOUNDS OF $\gamma(\lambda)$}

In this section, we first give an upper bound to the delaydistance ratio, $\gamma(\lambda)$. Then, we make further analysis on first passage delay and introduce a concept called cluster to cluster transmission. Using this concept, we derive a lower bound. Finally, we take propagation delay into consideration, and formulate its impact on $\gamma(\lambda)$.

\section{A. Upper Bound of $\gamma(\lambda)$}

Recall the definition of $\gamma(\lambda)$ (Eqn. 6) that $\gamma(\lambda)=$ $\lim _{d(x, y) \rightarrow \infty} \frac{T_{\lambda}(x, y)}{d(x, y)}$, where $x, y$ belongs to the giant component of $\mathcal{G}\left(\lambda, r_{0}, f(r)\right)$. To compute such a limit, we do not have to calculate $\gamma(\lambda)$ for all $x, y \in \mathcal{C}\left(\mathcal{G}\left(\lambda, r_{0}, f(r)\right)\right)$. The correctness of this assertion is assured by the following lemma.

Lemma 1. Given a convergent sequence $\left\{x_{k}\right\}, k=1,2, \ldots$, and $\lim _{k \rightarrow \infty} x_{k}=x_{0} .\left\{y_{k}\right\}, k=1,2, \ldots$, is a subsequence of $\left\{x_{k}\right\}$, and $\lim _{k \rightarrow \infty} y_{k}=y_{0}$. Then $x_{0}=y_{0}$.

It is easy to see that the number of nodes in $\mathcal{C}\left(\mathcal{G}\left(\lambda, r_{0}, f(r)\right)\right)$ is countable. We enumerate for all nodes. We randomly select a node and label it as $x_{0}$, and then label other nodes according to the distance from $x_{0}$ (larger subscript means larger distance from $x_{0}$ ). Define sequence $\left\{m_{k}, k=1,2, \ldots,\right\}, m_{k}=\frac{T_{\lambda}\left(x_{0}, x_{k}\right)}{d\left(x_{0}, x_{k}\right)}$, then $\lim _{k \rightarrow \infty} m_{k}=$ $\gamma(\lambda)$. According to lemma 1, we only need to find a subset of nodes of $\mathcal{C}\left(\mathcal{G}\left(\lambda, r_{0}, f(r)\right)\right)$ (the cardinality of this subset must be infinity), and calculate $\gamma(\lambda)$ from this subset. This technique is used in deriving the upper bound.

Now we are to obtain the upper bound of $\gamma(\lambda)$. The delay $T_{\lambda}(x, y)$ is defined as the minimum delay along all paths connecting nodes $x$ and $y$. Thus, it must be smaller than or equal to the delay along one specific path. In this part, we first find a subset of nodes of $\mathcal{C}\left(\mathcal{G}\left(\lambda, r_{0}, f(r)\right)\right)$. Then, we find a path for each node pair in this subset. After that, we calculate the delay along this path. Finally, dividing the delay by distance, we obtain an upper bound of $\gamma(\lambda)$.

Before proceeding, we need the following lemma.

Lemma 2. Consider Poisson Boolean models in $\mathbb{R}^{2}$. Let $\lambda_{c}(r)$ denote the critical density in the case where the transmission range is $r$. Then it is the case that

$$
\lambda_{c}\left(r_{1}\right) r_{1}^{2}=\lambda_{c}\left(r_{2}\right) r_{2}^{2} \text {, }
$$

where $r_{1}, r_{2}>0$.

Proof: See Proposition 2.10 in [15].

The long-term critical density $\lambda_{L}$ is also the critical density of Poisson Boolean Model with transmission range $r_{0}$. Consider the network with density $\lambda>\lambda_{L}$, according to lemma 2, we immediately know that when $\lambda r^{2}>\lambda_{L} r_{0}^{2}$, i.e., $r>\sqrt{\frac{\lambda_{L}}{\lambda}} \cdot r_{0}$, Poisson Boolean Model $\mathcal{B}(\lambda, r)$ is percolated.

Let $\tilde{r}=r_{0} \sqrt{\frac{\lambda_{L}(1+\epsilon)}{\lambda}}$, then $\mathcal{B}(\lambda, \tilde{r})$ is percolated. Further, since $\lambda \geq \lambda_{L}(1+\epsilon)$, we must have $\tilde{r} \leq r_{0}$. Note that, the Random Connection Model $\mathcal{G}\left(\lambda, r_{0}, f(r)\right)$ is essentially a Poisson Boolean Model $\mathcal{B}\left(\lambda, r_{0}\right)$ with $r_{0} \geq \tilde{r}$. Hence, $\mathcal{B}(\lambda, \tilde{r})$ is a subgraph of $\mathcal{G}\left(\lambda, r_{0}, f(r)\right)$. (Here, $\mathcal{B}(\lambda, \tilde{r})$ has the same node locations as $\mathcal{G}\left(\lambda, r_{0}, f(r)\right)$.) We denote the giant component of $\mathcal{B}(\lambda, \tilde{r})$ by $\mathcal{C}(\mathcal{B}(\lambda, \tilde{r}))$. According to the uniqueness of giant component in supercritical case, there must be $\mathcal{C}(\mathcal{B}(\lambda, \tilde{r})) \subseteq \mathcal{C}\left(\mathcal{G}\left(\lambda, r_{0}, f(r)\right)\right)$. According to lemma 1, when calculating $\gamma(\lambda)$, we only need to focus on the case that both nodes belong to $\mathcal{C}(\mathcal{B}(\lambda, \tilde{r}))$.

Assume that nodes $u, v \in \mathcal{C}(\mathcal{B}(\lambda, \tilde{r}))$. Then there exists at least one path in $\mathcal{B}(\lambda, \tilde{r})$ from $u$ to $v$. We choose the path with minimum number of hops, and denote it by $\pi_{m}$.

Up to now, we have found a path connecting $u$ and $v$. Next, we are to calculate the delay along this path. To start with, we need to compute the number of hops, denoted by $N_{\lambda}(d(u, v))$, in $\pi_{m}$. We can find a relationship between $N_{\lambda}(d(u, v))$ and $\lambda$ using the following scaling arguments.

Scale the network $\mathcal{B}(\lambda, \tilde{r})$ up by $\sqrt{\frac{\lambda}{\lambda_{L}}}$, then the network $\mathcal{B}(\lambda, \tilde{r})$ becomes another network $\mathcal{B}\left(\lambda_{L}, r_{0} \sqrt{1+\epsilon}\right)$. Further, the original distance $d(u, v)$ between $u$ and $v$ becomes $d(u, v) \sqrt{\frac{\lambda}{\lambda_{L}}}$. Then to compute $N_{\lambda}(d(u, v))$, it is equivalent to compute $N_{\lambda_{L}}\left(d(u, v) \sqrt{\frac{\lambda}{\lambda_{L}}}\right)$. Next, we present the lemma concerning $N_{\lambda_{L}}(d)$.

Lemma 3. Given $\mathcal{B}\left(\lambda_{L}, r_{0} \sqrt{1+\epsilon}\right)$, and $u, v \in$ $\mathcal{C}\left(\mathcal{B}\left(\lambda_{L}, r_{0} \sqrt{1+\epsilon}\right)\right)$, the minimal number of hops among all paths from $u$ to $v$ is $N_{\lambda_{L}}(d(u, v))$. Then there exist $\kappa_{\epsilon}$ such that

$$
\lim _{d(u, v) \rightarrow \infty} \frac{N_{\lambda_{L}}(d(u, v))}{d(u, v)}=\kappa_{\epsilon} .
$$


Lemma 3 gives the rigorous definition of $\kappa_{\epsilon}$, which is a useful parameter in Theorem 2 and Theorem 3. The proof of Lemma 3 is based on a conclusion on subadditivity and is given in section V-A.

According to lemma 3 , we immediately get

$$
\begin{aligned}
\lim _{d(u, v) \rightarrow \infty} \frac{N_{\lambda}(d(u, v))}{d(u, v)} & =\lim _{d(u, v) \rightarrow \infty} \frac{N_{\lambda_{L}}\left(d(u, v) \sqrt{\frac{\lambda}{\lambda_{L}}}\right)}{d(u, v)} \\
& =\kappa_{\epsilon} \sqrt{\frac{\lambda}{\lambda_{L}}} .
\end{aligned}
$$

Then we calculate the delay $T_{p}\left(\pi_{m}\right)$ along path $\pi_{m}$. According to the Strong Law of Large Numbers, with probability 1, we have

$\lim _{d(u, v) \rightarrow \infty} \frac{T_{p}\left(\pi_{m}\right)}{N_{\lambda}(d(u, v))}=\lim _{d(u, v) \rightarrow \infty} \frac{\sum_{e \in \pi_{m}} T_{c}(e)}{N_{\lambda}(d(u, v))}=E\left[T_{c}(e)\right]$.

Therefore,

$$
\begin{aligned}
\gamma(\lambda) & =\lim _{d \rightarrow \infty} \frac{T_{\lambda}(u, v)}{d(u, v)} \leq \lim _{d \rightarrow \infty} \frac{T_{p}\left(\pi_{m}\right)}{d(u, v)} \\
& =\lim _{d(u, v) \rightarrow \infty} \frac{T_{p}\left(\pi_{m}\right)}{N_{\lambda}(d(u, v))} \cdot \lim _{d \rightarrow \infty} \frac{N_{\lambda}(d(u, v))}{d(u, v)} \\
& =\kappa_{\epsilon} \sqrt{\frac{\lambda}{\lambda_{L}}} E\left[T_{c}(e)\right] .
\end{aligned}
$$

From the definition of the path $\pi_{m}$, we know that the length of each hop is smaller than $\tilde{r}=r_{0} \sqrt{\frac{\lambda_{L}(1+\epsilon)}{\lambda}}$. Besides, the connection function $g(r)$ is monotone decreasing. Thus, for a link $e^{\prime}$ whose length is $\tilde{r}$, there must be

$$
\begin{aligned}
E\left[T_{c}(e)\right] & \leq E\left[T_{c}\left(e^{\prime}\right)\right]=\sum_{k=0}^{\infty} k \mathbb{P}\left(T_{c}\left(e^{\prime}\right)=k\right) \\
& =\sum_{k=0}^{\infty} k(1-g(\tilde{r}))^{k} g(\tilde{r})=\frac{1}{g(\tilde{r})}-1 .
\end{aligned}
$$

Thus,

$$
\begin{aligned}
\gamma(\lambda) & =\kappa_{\epsilon} \sqrt{\frac{\lambda}{\lambda_{L}}} E\left[T_{c}(e)\right] \leq \kappa_{\epsilon} \sqrt{\frac{\lambda}{\lambda_{L}}}\left(\frac{1}{g(\tilde{r})}-1\right) \\
& =\kappa_{\epsilon} \sqrt{\frac{\lambda}{\lambda_{L}}}\left(\frac{1}{g\left(r_{0} \sqrt{\frac{\lambda_{L}(1+\epsilon)}{\lambda}}\right)}-1\right) .
\end{aligned}
$$

Furthermore, from property (2) of theorem 1, we know $\gamma(\lambda)$ is a monotone decreasing function. Thus,

$$
\gamma(\lambda) \leq \inf _{\lambda^{\prime} \in\left[\lambda_{L}(1+\epsilon), \lambda\right]} \kappa \sqrt{\frac{\lambda^{\prime}}{\lambda_{L}}}\left(\frac{1}{g\left(r_{0} \sqrt{\frac{\lambda_{L}(1+\epsilon)}{\lambda^{\prime}}}\right)}-1\right) .
$$

\section{B. Lower Bound of $\gamma(\lambda)$}

1) Cluster to Cluster Transmission: In section IV-A, we have obtained the upper bound of $\gamma(\lambda)$ by calculating the delay along one path. However, the method used in section IV-A cannot be used to study the lower bound of $\gamma(\lambda)$. It is hard to obtain the number of paths connecting two nodes. The delay along each path is random and may correlate with that of other paths. Therefore, it is usually intractable to obtain the delay from Eqn. (4). To overcome this difficulty, we introduce the concept of cluster to cluster transmission.

Consider the packet transmission process from node $u$ to node $v$. Assume that at time slot $t_{1}$, node $u_{1}\left(u_{1}=u\right)$ transmits a packet to other nodes. Since we have ignored the propagation delay, all nodes connected to $u_{1}$ in $\mathcal{G}_{t_{1}}\left(\lambda, r_{0}, g(r)\right)$, denoted by $W_{1}$, receive the information instantaneously. Then, the transmission process stops. This is because all outgoing connections from $W_{1}$ are unavailable at this time instance. The transmission process will restart at time slot $t_{2}>t_{1}{ }^{7}$, when at least one node in $W_{1}$ find the opportunity to forward the packet to a new node, denoted by $u_{2}$. At this time slot, $u_{2}$ transmits the packet to a set of new nodes (not in $W_{1}$ ) which are connected to $u_{2}$ in $\mathcal{G}_{t_{2}}\left(\lambda, r_{0}, g(r)\right)$, denoted by $W_{2}$, instantaneously. This process goes on, until at time slot $t_{M}$, node $u_{M}$ and the destination node $v$ are in the same connected cluster and the packet is transmitted to node $v$ instantaneously.

We can see that the cluster to cluster transmission as a series of outbursts. During each outburst, a set of new nodes receive the packet. $W_{k}, k=1,2, \ldots, M$ is the set of nodes which receive the information right at the $k$ th outburst. $u_{k} \in W_{k-1}$ is the starting node at the $k$ th outburst. It is possible that at time $t_{k}$, two nodes $u_{k}^{1}$ and $u_{k}^{2}$ find transmission opportunity simultaneously. Choosing different starting nodes will lead to different cluster to cluster transmission processes.

A cluster to cluster transmission process can be fully described by $\Pi=\left\{\left(t_{1}, u_{1}\right),\left(t_{2}, u_{2}\right), \ldots,\left(t_{M}, u_{M}\right)\right\}$. Packets can be transmitted from $u$ to $v$ through $\Pi$. Define the passage time for the cluster to cluster transmission process $\Pi$ as

$$
T_{p}(\Pi)=t_{M}-t_{1} .
$$

Then, we have the following lemma.

Lemma 4. Given nodes $u, v \in \mathcal{C}\left(\mathcal{G}\left(\lambda, r_{0}, f(r)\right)\right)$, the first passage time

$$
\begin{array}{r}
T_{\lambda}(u, v)=\inf \left\{T_{p}(\Pi) \mid \Pi\right. \text { is a cluster to cluster } \\
\text { transmission process from } u \text { to } v\} .
\end{array}
$$

Proof: For convenience, we use $\mathscr{L}$ to denote the set of all the cluster to cluster transmission processes from $u$ to $v$. Then Eqn.(12) can be rewritten as

$$
T_{\lambda}(u, v)=\inf \left\{T_{p}(\Pi) \mid \Pi \in \mathscr{L}\right\} .
$$

It is easy to see that for each cluster to cluster transmission process $\Pi$ from $u$ to $v$,

$$
T_{p}(\Pi) \geq T_{\lambda}(u, v) .
$$

Thus,

$$
\inf \left\{T_{p}(\Pi) \mid \Pi \in \mathscr{L}\right\} \geq T_{\lambda}(u, v) .
$$

Next, we show that

$$
\inf \left\{T_{p}(\Pi) \mid \Pi \in \mathscr{L}\right\} \leq T_{\lambda}(u, v) .
$$

\footnotetext{
${ }^{7}$ Here, we do not require $t_{2}$ to be the smallest, i.e., there may exist $t_{1}<$ $t^{\prime}<t_{2}$, such that at least one node in $w_{1}$ have the opportunity to forward the information to a new node at time slot $t^{\prime}$.
} 
Recall the definition of $T_{\lambda}(u, v)$, i.e.,

$$
T_{\lambda}(u, v)=\inf \left\{T_{p}(\pi): \pi \text { is a path from } u \text { to } v\right\} .
$$

Let $\pi_{0}$ be the path with minimum delay from $u$ to $v$, we prove that there exists a cluster to cluster transmission process $\Pi_{0}$, such that $T_{p}\left(\Pi_{0}\right)=T_{p}\left(\pi_{0}\right)$.

Assume that $\pi_{0}=i_{0} i_{1} i_{2} \ldots i_{K}\left(i_{0}=u, i_{K}=v\right)$. At time slot $t_{1}$, some nodes in path $\pi_{0}$ may be in the same connected cluster as $i_{0}$. Let $i_{\eta_{1}-1}$ be the node attainable from $i_{0}$ with largest subindex, then the link between $i_{\eta_{1}-1}$ and $i_{\eta_{1}}$ must be off at this time slot. Let $t_{2}>t_{1}$ be the first time slot that this link is on. At time slot $t_{2}$, let $i_{\eta_{2}-1}$ be the node attainable from $i_{\eta_{1}}$ with largest subindex. Then the link between $i_{\eta_{2}-1}$ and $i_{\eta_{2}}$ must be off until time slot $t_{3}>t_{2} \ldots$ At time slot $t_{k}$, node $i_{\eta_{k-1}}$ and destination node $v$ are in the same connected cluster and the information transmit to $v$ instantaneously. We denote by $\Pi_{0}$ this cluster to cluster transmission process. And

$$
\Pi_{0}=\left\{\left(t_{1}, i_{0}\right),\left(t_{2}, i_{\eta_{1}}\right), \ldots,\left(t_{k}, i_{\eta_{k-1}}\right)\right\} .
$$

From the construction of $\Pi_{0}$, it is obvious that $T_{p}\left(\Pi_{0}\right)=$ $T_{p}\left(\pi_{0}\right)$.

Thus,

$$
\begin{aligned}
T_{\lambda}(u, v) & =T_{p}\left(\pi_{0}\right)=T_{p}\left(\Pi_{0}\right) \\
& \geq \inf \left\{T_{p}(\Pi) \mid \Pi \in \mathscr{L}\right\} .
\end{aligned}
$$

Combining Eqn.(21) and Eqn.(14), we obtain

$$
T_{\lambda}(u, v)=\inf \left\{T_{p}(\Pi) \mid \Pi \in \mathscr{L}\right\} .
$$

Based on the cluster to cluster transmission process, we are now ready to compute a lower bound of $\gamma(\lambda)$.

2) Compute the Lower Bound: In this section, we use the concept of cluster to cluster transmission to derive a lower bound of $\gamma(\lambda)$.

To start with, we need to introduce a random variable $S_{g, t, u}(\lambda)$ ( $g$ is the connection function, $u$ is a node) to represent the radius of the connected cluster $W(\{u\})$ in the instantaneous geometric random graph $\mathcal{G}_{t}\left(\lambda, r_{0}, g(r)\right)$. We establish a cartesian coordinate in $\mathbb{R}^{2}$. We define $H\left(z_{0}, a\right)$ as

$$
H\left(z_{0}, a\right)=\left\{z=\left(z_{x}, z_{y}\right) \in \mathbb{R}^{2} \mid\left\|z-z_{0}\right\|<a\right\} .
$$

The random variable $S_{g, t, u}(\lambda)$ is defined as

$S_{g, t, u}(\lambda)=\sup \{a \mid \exists$ node $v \in H(u, a), v \leftrightarrow u$ at time slot $t\}$.

According to the spacial stationarity and time independence of our dynamic RCM $\mathcal{G}_{t}\left(\lambda, r_{0}, g(r)\right)$, the distribution of $S_{g, t, u}(\lambda)$ is independent of $t$ and $u$. Thus, we can write $S_{g, t, u}(\lambda)$ as $S_{g}(\lambda)$ if causing no confusion.

Now, given two nodes $u$ (source) and $v$ (destination), consider a cluster to cluster transmission process(Fig. 2)

$$
\Pi=\left\{\left(u^{(1)}, t_{1}\right),\left(u^{(2)}, t_{2}\right), \ldots,\left(u^{(M)}, t_{M}\right)\right\},
$$

where $u^{(1)}=u$, and $u^{(M)}, v$ are in the same connected cluster at the time instance $t_{M}$. Then the delay along this cluster to cluster transmission process is

$$
T_{p}(\Pi)=t_{M}-t_{1}=\sum_{k=1}^{M-1}\left(t_{k+1}-t_{k}\right) \geq M-1 .
$$

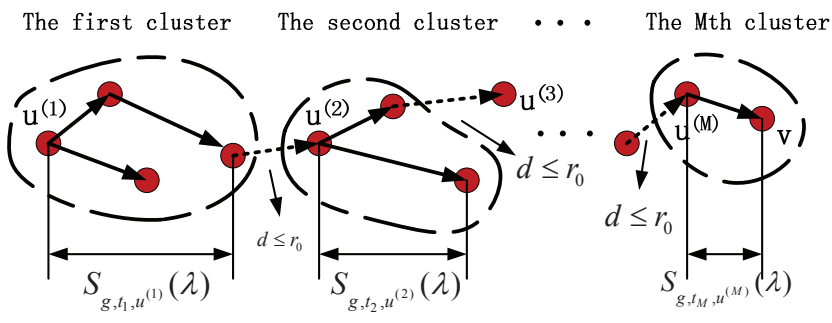

Fig. 2. Illustration of a cluster to cluster transmission process.

Note that, $\forall k=1,2, \ldots, M-1, u^{(k+1)}$ is connected to a node in $W_{k}$, denoted by $u^{\prime}$. Then,

$$
\begin{aligned}
\left\|u^{(k+1)}-u^{(k)}\right\| & \leq\left\|u^{(k+1)}-u^{\prime}\right\|+\left\|u^{\prime}-u^{(k)}\right\| \\
& \leq S_{g, t_{k}, u^{(k)}}(\lambda)+r_{0} .
\end{aligned}
$$

As for $k=M$,

$$
\left\|v_{x}-u^{(M)}\right\| \leq S_{g, t_{M}, u^{(M)}}(\lambda) .
$$

Combining the above two inequalities together, we obtain,

$$
\begin{aligned}
d(u, v) & =\|v-u\| \\
& \leq \sum_{k=1}^{M-1}\left\|u^{(k+1)}-u^{(k)}\right\|+\left\|v-u^{(M)}\right\| \\
& \leq \sum_{k=1}^{M-1}\left(S_{g, t_{k}, u^{(k)}}(\lambda)+r_{0}\right)+S_{g, t_{M}, u_{x}^{(M)}}(\lambda) \\
& <\sum_{k=1}^{M}\left(S_{g, t_{k}, u^{(k)}}(\lambda)+r_{0}\right) .
\end{aligned}
$$

For each $k$, it is easy to check that $S_{g, t_{k}, u^{(k)}}(\lambda)$ admits the same distribution as another random variable $S_{g}(\lambda)$. Further, we can prove the following lemma.

Lemma 5. With probability 1 , we have

$$
\lim _{M \rightarrow \infty} \frac{\sum_{k=1}^{M} S_{g, t_{k}, u^{(k)}}(\lambda)}{M}=E\left(S_{g}(\lambda)\right) .
$$

We need to emphasize that Lemma 5 cannot be obtained directly from the Strong Law of Large Number, because $S_{g, t_{k}, u^{(k)}}(\lambda)$ 's may not be independent for different $k$ 's. However, we can show that the random process $\left\{S_{g, t_{k}, u^{(k)}}(\lambda)\right\}_{k}$ is in fact ergodic. Then, according to Birkhoff Ergodic Theorem, the time average of $S_{g, t_{k}, u^{(k)}}(\lambda)$ (left hand side of Eqn. (16)) will be equal to the spacial average of $S_{g, t_{k}, u^{(k)}}(\lambda)$ (right hand side of Eqn. (16)). See Section V-E for the detailed proof.

According to Lemma 5, $\forall \epsilon_{1}>0, \exists M_{\epsilon_{1}}$, such that $\forall M>$ $M_{\epsilon_{1}}$ (this condition is satisfied for large enough $d(u, v)$ ), we have

$$
\frac{\sum_{k=1}^{M}\left(S_{g, t_{k}, u^{(k)}}(\lambda)+r_{0}\right)}{M}<E\left(S_{g}(\lambda)+r_{0}\right)+\epsilon_{1} .
$$

Combined with Eqn. (15), we have

$$
d(u, v)<M\left(E\left(S_{g}(\lambda)+r_{0}\right)+\epsilon_{1}\right) .
$$


Then

$$
T_{p}(\Pi) \geq M-1>\frac{d(u, v)}{E\left(S_{g}(\lambda)+r_{0}\right)+\epsilon_{1}}-1 .
$$

Note that the right hand side of the above inequality does not depend on the selection of the cluster to cluster transmission processes. Thus,

$$
T_{\lambda}(u, v) \geq \frac{d(u, v)}{E\left(S_{g}(\lambda)+r_{0}\right)+\epsilon_{1}}-1 .
$$

Therefore,

$$
\begin{aligned}
\gamma(\lambda) & =\lim _{d(u, v) \rightarrow \infty} \frac{T_{\lambda}(u, v)}{d(u, v)} \\
& \geq \frac{1}{E\left(S_{g}(\lambda)+r_{0}\right)+\epsilon_{1}}
\end{aligned}
$$

Let $\epsilon_{1} \rightarrow 0$, we finally obtain

$$
\gamma(\lambda) \geq \frac{1}{E\left(S_{g}(\lambda)+r_{0}\right)} .
$$

\section{Impact of Propagation Delay}

The delay in large-scale Wireless Networks is composed of two parts, i.e., the waiting delay and the propagation delay. In previous sections, we ignored the propagation delay $\tau$. Now, we will consider its impact on $\gamma(\lambda)$.

The propagation delay will not have much influence on $\gamma(\lambda)$ when $\lambda$ is relatively small, because the first passage delay is dominated by the waiting delay. However, when $\lambda$ is relatively large, e.g., $\lambda>\lambda_{I}$, the network connectivity is maintained at each time slot, and thus the propagation delay will increase $\gamma(\lambda)$ substantially $(\gamma(\lambda)$ is now bounded below away from 0$)$.

The proof of Theorem 3 is in fact a generalization of that of Theorem 2.

Proof: We first consider the upper bound. We have already obtained Eqn. (10), i.e.,

$$
\gamma(\lambda) \leq \kappa_{\epsilon} \sqrt{\frac{\lambda}{\lambda_{L}}} E\left[T_{c}(e)\right]
$$

where the length of link $e$ is smaller than $\tilde{r}=r_{0} \sqrt{\frac{\lambda_{L}(1+\epsilon)}{\lambda}}$.

Using similar method in deriving Eqn. (11), we obtain

$$
\begin{aligned}
E\left[T_{c}(e)\right] & \leq E\left[T_{c}\left(e^{\prime}\right)\right]=\sum_{k=0}^{\infty}(k+\tau) \mathbb{P}\left(T_{c}\left(e^{\prime}\right)=k\right) \\
& <\sum_{k=0}^{\infty}(k+1)(1-g(\tilde{r}))^{k} g(\tilde{r})=\frac{1}{g(\tilde{r})} \\
& =\frac{1}{g\left(r_{0} \sqrt{\frac{\lambda_{L}(1+\epsilon)}{\lambda}}\right)}
\end{aligned}
$$

where $e^{\prime}$ is link whose length is $\tilde{r}$. The inequality above is slightly different from Eqn. (11). This is because we have taken propagation delay into consideration.

Thus,

$$
\gamma(\lambda) \leq \kappa_{\epsilon} \sqrt{\frac{\lambda}{\lambda_{L}}} \frac{1}{g\left(r_{0} \sqrt{\frac{\lambda_{L}(1+\epsilon)}{\lambda}}\right)} .
$$

Note that $\gamma(\lambda)$ is a monotone decreasing function, thus

$$
\gamma(\lambda) \leq \inf _{\lambda^{\prime} \in\left[\lambda_{L}(1+\epsilon), \lambda\right]} \kappa_{\epsilon} \sqrt{\frac{\lambda^{\prime}}{\lambda_{L}}} \frac{1}{g\left(r_{0} \sqrt{\frac{\lambda_{L}(1+\epsilon)}{\lambda^{\prime}}}\right)} .
$$

Then we consider the lower bound. Similar to the previous part, we still focus on the cluster to cluster transmission. Consider a cluster to cluster transmission process

$$
\Pi=\left\{\left(u^{(1)}, t_{1}\right),\left(u^{(2)}, t_{2}\right), \ldots,\left(u^{(M)}, t_{M}\right)\right\} .
$$

Similarly, we have, $\forall k=1,2, \ldots, M-1$,

$$
\left\|u^{(k+1)}-u^{(k)}\right\| \leq S_{g, t_{k}, u^{(k)}}(\lambda)+r_{0} .
$$

As for $k=M$,

$$
\left\|v-u^{(M)}\right\| \leq S_{g, t_{M}, u_{x}^{(M)}}(\lambda)<S_{g, t_{M}, u^{(M)}}(\lambda)+r_{0} .
$$

Besides, the distance transmitted is also limited by the finite hops in one time slot. Since each hop takes $\tau$ fraction of a time slot, then a packet can transmit at most $\frac{1}{\tau}$ hops in one time slot. As a result, the longest distance transmitted in one time slot is upper bounded by $\frac{r_{0}}{\tau}$. Then $\forall k=1,2, \ldots, M-1$,

$$
\left\|u^{(k+1)}-u^{(k)}\right\| \leq \frac{r_{0}}{\tau}+r_{0}
$$

As for $k=M$,

$$
\left\|v-u^{(M)}\right\| \leq \frac{r_{0}}{\tau}<\frac{r_{0}}{\tau}+r_{0} .
$$

Integrating the above four inequalities, we obtain, $\forall k=$ $1,2, \ldots, M-1$,

$$
\left\|u^{(k+1)}-u^{(k)}\right\| \leq \min \left\{S_{g, t_{k}, u^{(k)}}(\lambda), \frac{r_{0}}{\tau}\right\}+r_{0} .
$$

As for $k=M$,

$$
\left\|v-u^{(M)}\right\| \leq \min \left\{S_{g, t_{M}, u^{(M)}}(\lambda), \frac{r_{0}}{\tau}\right\}+r_{0} .
$$

Again, using the method in section IV-B, we immediately obtain

$$
\gamma(\lambda) \geq \frac{1}{E\left(\min \left\{S_{g}(\lambda), \frac{r_{0}}{\tau}\right\}\right)+r_{0}} .
$$

\section{PROOF}

\section{A. Proof of Lemma 3}

The method used in this proof is similar to that used by Dousse et al. in [11] and Kong et al. in [12].

We first construct a cartesian coordinate system. Without loss of generality, we assume that there is a node at the origin. Let $z_{n}=\arg \min _{z \in \mathcal{C}\left(\mathcal{B}\left(\lambda_{L}, r_{0} \sqrt{1+\epsilon}\right)\right.}\{d(z,(0, n))\}$. We define $N_{\lambda_{L}}^{\prime}(m, n)=N_{\lambda_{L}}\left(d\left(z_{n}, z_{m}\right)\right)$. We first prove that $N_{\lambda_{L}}^{\prime}(0, m)$ scales linearly with respect to $m$ (Lemma 6). As we will see later, Lemma 3 follows directly from Lemma 6.

Lemma 6. There exists $\kappa_{\epsilon}$, such that

$$
\lim _{m \rightarrow \infty} \frac{N_{\lambda_{L}}^{\prime}(0, m)}{m}=\kappa_{\epsilon} .
$$

To prove Lemma 6, we use Liggett's subadditive ergodic theorem, which is formally restated below. 
Lemma 7. (Liggett [16])Let $\left\{S_{l, m}\right\}$ be a collection of random variables indexed by integers $0 \leq l \leq m$. Suppose $\left\{S_{l, m}\right\}$ has the following properties:

1) $S_{0, m} \leq S_{0, l}+S_{l, m}, 0 \leq l \leq m$;

2) $\left\{S_{(m-1) k, m k}, m \geq 1\right\}$ is a stationary process for each $k$;

3) $\left\{S_{l, l+k}, k \geq 0\right\}=\left\{S_{l+1, l+k+1}, k \geq 0\right\}$ in distribution for each l;

4) $E\left[\left|S_{0, m}\right|\right]<\infty$ for each $m$.

Then $\alpha \triangleq \lim _{m \rightarrow \infty} \frac{E\left[S_{0, m}\right]}{m}=\inf _{m \geq 1} \frac{E\left[S_{0, m}\right]}{m} ; S \triangleq$ $\lim _{m \rightarrow \infty} \frac{S_{0, m}}{m}$ exists with probability 1 and $E[S]=\alpha$. Furthermore, if

5. the stationary process $\left\{S_{(m-1) k, m k}, m \geq 1\right\}$ is ergodic; then $S=\alpha$ with probability 1 .

It is easy to see that $N_{\lambda_{L}}^{\prime}(0, m) \leq N_{\lambda_{L}}^{\prime}(0, l)+$ $N_{\lambda_{L}}^{\prime}(l, m)(0 \leq l \leq m)$. Then the first condition of lemma 7 is satisfied. Since Poisson Boolean Model $\mathcal{B}\left(\lambda_{L}, r_{0} \sqrt{1+\epsilon}\right)$ is homogeneous, the second and the third conditions of lemma 7 are also satisfied. Now, we only need to prove that conditions 4 and 5 are also satisfied.

Lemma 8. $E\left(N_{\lambda_{L}}^{\prime}(0, m)\right)<\infty$.

Note that $E\left(N_{\lambda_{L}}^{\prime}(0, m)\right)=\sum_{i=1}^{\infty} \mathbb{P}\left(N_{\lambda_{L}}^{\prime}(0, m) \geq i\right)$. If we can show that $\mathbb{P}\left(N_{\lambda_{L}}^{\prime}(0, m) \geq i\right)$ decays exponentially with respect to $i$, then Lemma 8 follows directly. The basic idea is to find a sequence of toruses circling nodes $z_{0}$ and $z_{m}$ using nodes in the giant component of $\mathcal{B}\left(\lambda_{L}, r_{0} \sqrt{1+\epsilon}\right)$ ). Then, we can show that the least number of hops $N_{\lambda_{L}}^{\prime}(0, m)$ from $z_{0}$ to $z_{m}$ cannot be arbitrarily large (see Fig. 5). Due to the supercriticality of $\mathcal{B}\left(\lambda_{L}, r_{0} \sqrt{1+\epsilon}\right)$ ), we can show that such toruses exist with probability close to 1 . Therefore, the probability that $N_{\lambda_{L}}^{\prime}(0, m)$ is greater than a large threshold is close to 0 . The detailed proof is provided in Section V-B.

Then condition 4 of lemma 7 is satisfied. Next, we prove that $N_{\lambda_{L}}^{\prime}(m, n)$ satisfies condition 5. To demonstrate that $N_{\lambda_{L}}^{\prime}(m k,(m+1) k)$ is ergodic, we show that it is strong mixing, which is a stronger property.

Lemma 9. $N_{\lambda_{L}}^{\prime}(m k,(m+1) k)$ is strong mixing.

The strong mixing property essentially states that the dependence between $N_{\lambda_{L}}^{\prime}(m k,(m+1) k)$ and $N_{\lambda_{L}}^{\prime}((m+n) k,(m+$ $n+1) k$ ) becomes negligibly small as $n$ becomes large. The strategy to prove this statement resembles some parts of the proof of Lemma 8. We first find two toruses circling the shortest path from $z_{m k}$ to $z_{(m+1) k}$ and the shortest path from $z_{(m+n) k}$ to $z_{(m+n+1) k}$, respectively. By the stationarity of Poisson Point Process, we know that the size of the two toruses do not depend on $n$. Hence, as $n$ increases, the two toruses will become disjoint eventually. By the spatial independence of Poisson Point Process, we can see that $N_{\lambda_{L}}^{\prime}(m k,(m+1) k)$ and $N_{\lambda_{L}}^{\prime}((m+n) k,(m+n+1) k)$ becomes approximately independent when $n$ is large enough. The detailed proof is provided in Section V-C.

Now, we have proved that $N_{\lambda_{L}}^{\prime}(m, n)$ satisfy conditions $1-5$ of Lemma 7. Thus, there exists $\kappa_{\epsilon}$, such that

$$
\lim _{m \rightarrow \infty} \frac{N_{\lambda_{L}}^{\prime}(0, m)}{m}=\kappa_{\epsilon} .
$$

Before proving Lemma 3, we also need the following lemma.

Lemma 10. $d\left(z_{n},(0, n)\right)<\infty$ with probability 1 .

Intuitively, if $d\left(z_{n},(0, n)\right)=\infty$, there does not exist any giant component in $\mathcal{B}\left(\lambda_{L}, r_{0} \sqrt{1+\epsilon}\right)$ ) (otherwise, $\left.d\left(z_{n},(0, n)\right)<\infty\right)$. This contradict to the supercriticality of $\left.\mathcal{B}\left(\lambda_{L}, r_{0} \sqrt{1+\epsilon}\right)\right)$. The detailed proof is presented in section $\mathrm{V}-\mathrm{D}$.

Now, we are ready to prove Lemma 3.

Proof: Consider $N_{\lambda_{L}}(d(u, v))$. Without loss of generality, we suppose that $u$ is at the origin, $v$ is at the $+x$ axis. Assume that integer $n_{d}$ satisfy $n_{d} \leq d(u, v)<n_{d}+1$, then

$$
N_{\lambda_{L}}(d(u, v)) \leq N_{\lambda_{L}}\left(d\left(0, z_{n_{d}}\right)\right)+N_{\lambda_{L}}\left(d\left(z_{n_{d}}, v\right)\right),
$$

and

$$
N_{\lambda_{L}}(d(u, v)) \geq N_{\lambda_{L}}\left(d\left(0, z_{n_{d}}\right)\right)-N_{\lambda_{L}}\left(d\left(z_{n_{d}}, v\right)\right) .
$$

Note that

$$
\begin{aligned}
d\left(z_{n_{d}}, v\right) & \leq d\left(z_{n_{d}},\left(0, n_{d}\right)\right)+d\left(\left(0, n_{d}\right), v\right) \\
& \leq d\left(z_{n_{d}},\left(0, n_{d}\right)\right)+1 \\
& <\infty .
\end{aligned}
$$

Using similar method in the proof of Lemma 8, we can prove $E\left(N_{\lambda_{L}}\left(d\left(z_{n_{d}}, v\right)\right)\right)<\infty$ (to avoid verbosity, we do not elaborate it here). Therefore, $N_{\lambda_{L}}\left(d\left(z_{n_{d}}, v\right)\right)<\infty$ with probability 1. Further, by the stationarity of Poisson Point Process, $N_{\lambda_{L}}\left(d\left(z_{n_{d}}, v\right)\right)$ does not depend on $d(u, v)$. Then, divide Eqn. (20) and Eqn. (21) by $d(u, v)$, and let $d(u, v) \rightarrow \infty$. We immediately obtain

$$
\lim _{d(u, v) \rightarrow \infty} \frac{N_{\lambda_{L}}(d(u, v))}{d(u, v)}=\kappa_{\epsilon} .
$$

\section{B. Proof of Lemma 8}

Proof: Consider $N_{\lambda_{L}}^{\prime}(0, m)$. Let $z^{\prime}=\frac{z_{0}+z_{m}}{2}$. We draw a series of squares centering at $z^{\prime}$ (Fig. 3), and the side lengths are $1,2,4, \ldots, 2^{k}, \ldots$.

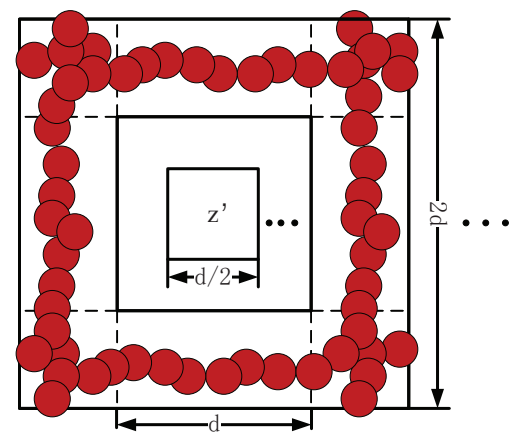

Fig. 3. A series of squares centering at $z^{\prime}$.

We use $R(d)$ to denote the rectangle with side lengths $\frac{d}{2}$ and $2 d$. We say $R(d)$ is good if and only if there exists a crossing connecting the two short sides in $R(d)$ (Fig. 4.(a)). We denote 
the event that $R(d)$ is good by $A_{R}(d)$. Note that the Poisson Boolean Model $\mathcal{B}\left(\lambda_{L}, r_{0} \sqrt{1+\epsilon}\right)$ is percolated. By Corollary 4.1 in [15], we have

$$
\lim _{d \rightarrow \infty} \mathbb{P}\left(A_{R}(d)\right)=1
$$

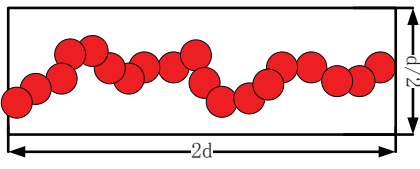

(a) Illustration of a good rectangle (b) Illustration of a good square $R(d)$.

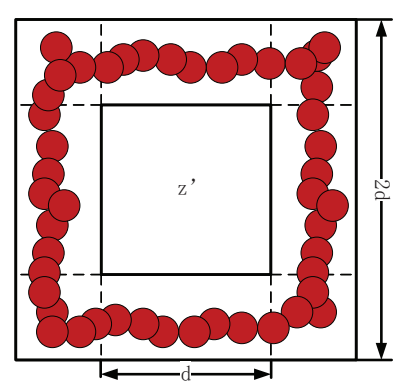

torus $C(d)$. Here, $z^{\prime}=\left(z_{x}^{\prime}, z_{y}^{\prime}\right)$.
Fig. 4. Illustration of a good rectangle and a good square torus.

We use $C(d)$ to denote the square torus $\left(\left[z_{x}^{\prime}-d, z_{x}^{\prime}+d\right] \times\right.$ $\left.\left[z_{y}^{\prime}-d, z_{y}^{\prime}+d\right]\right) \backslash\left(\left[z_{x}^{\prime}-\frac{d}{2}, z_{x}^{\prime}+\frac{d}{2}\right] \times\left[z_{y}^{\prime}-\frac{d}{2}, z_{y}^{\prime}+\frac{d}{2}\right]\right)$. We say $C(d)$ is good if and only if there exists a circuit in $C(d)$ (Fig. 4.(b)). We denote the event that $C(d)$ is good by $A_{C}(d)$. From Fig. 4.(b), we can see that $C(d)$ is composed of four $\frac{d}{2} \times 2 d$ rectangles, i.e., $R_{1}(d)=\left[z_{x}^{\prime}-d, z_{x}^{\prime}-\frac{d}{2}\right] \times\left[z_{y}^{\prime}-d, z_{y}^{\prime}+d\right]$, $R_{2}(d)=\left[z_{x}^{\prime}+\frac{d}{2}, z_{x}^{\prime}+d\right] \times\left[z_{y}^{\prime}-d, z_{y}^{\prime}+d\right], R_{3}(d)=\left[z_{x}^{\prime}-d, z_{x}^{\prime}+\right.$ $d] \times\left[z_{y}^{\prime}+\frac{d}{2}, z_{y}^{\prime}+d\right], R_{4}(d)=\left[z_{x}^{\prime}-d, z_{x}^{\prime}+d\right] \times\left[z_{y}^{\prime}-d, z_{y}^{\prime}-\frac{d}{2}\right]$. And we use $A_{R_{i}}(d)(i=1,2,3,4)$ to represent the event that $R_{i}(d)$ is good.

Obviously, if $\forall i=1,2,3,4, R_{i}(d)$ is good, $C(d)$ must be good. Thus,

$$
\mathbb{P}\left(A_{C}(d)\right) \geq \mathbb{P}\left(A_{R_{1}}(d) \bigcap A_{R_{2}}(d) \bigcap A_{R_{3}}(d) \bigcap A_{R_{4}}(d)\right) .
$$

Note that $\forall i=1,2,3,4, A_{R_{i}}(d)$ is an increasing event ${ }^{8}$. According to the FKG Inequality (Theorem 2.4 in [10]), we have

$$
\begin{aligned}
\mathbb{P}\left(A_{C}(d)\right) & \geq \mathbb{P}\left(A_{R_{1}}(d) \bigcap A_{R_{2}}(d) \bigcap A_{R_{3}}(d) \bigcap A_{R_{4}}(d)\right) \\
& \geq \mathbb{P}\left(A_{R_{1}}(d)\right) \mathbb{P}\left(A_{R_{2}}(d)\right) \mathbb{P}\left(A_{R_{3}}(d)\right) \mathbb{P}\left(A_{R_{4}}(d)\right) \\
& =\mathbb{P}\left(A_{R}(d)\right)^{4} .
\end{aligned}
$$

Combine Eqn. (22) with Eqn. (23), and we obtain

$$
\lim _{d \rightarrow \infty} \mathbb{P}\left(A_{C}(d)\right)=1 .
$$

Thus, $\forall \frac{1}{2}<\rho<1$, there exists $d_{\rho}$, such that, $\forall d \geq d_{0}$,

$$
\mathbb{P}\left(A_{C}(d)\right) \geq \rho .
$$

Let $k_{0}=\min \left\{k \mid 2^{k} \geq\left\|z_{m}-z_{0}\right\|, 2^{k} \geq d_{\rho}\right\}$, then for all $k \geq k_{0}$,

$$
\mathbb{P}\left(A_{C}\left(2^{k}\right)\right) \geq \rho .
$$

Assume that $C\left(2^{k}\right)\left(k \geq k_{0}\right)$ is good, if the shortest path from $z_{0}$ to $z_{m}$ is not contained in the square $\left[z_{x}^{\prime}-2^{k}, z_{x}^{\prime}+2^{k}\right] \times$

\footnotetext{
${ }^{8} \mathrm{~A}$ random variable $N$ is on the measurable pair $(\Omega, \mathscr{F})$ is called increasing if $N(\omega) \leq N\left(\omega^{\prime}\right)$ whenever $\omega \leq \omega^{\prime}$.
}

$\left[z_{y}^{\prime}-2^{k}, z_{y}^{\prime}+2^{k}\right]$, it must intersect with the circuit in $C\left(2^{k}\right)(\mathrm{Fig}$ $5)$. We can replace the part of the path $A C B$ with $A D B$, then the resulting path is shorter. Thus, the shortest path from $z_{0}$ to $z_{m}$ must be contained in $\left[z_{x}^{\prime}-2^{k}, z_{x}^{\prime}+2^{k}\right] \times\left[z_{y}^{\prime}-2^{k}, z_{y}^{\prime}+2^{k}\right]$.

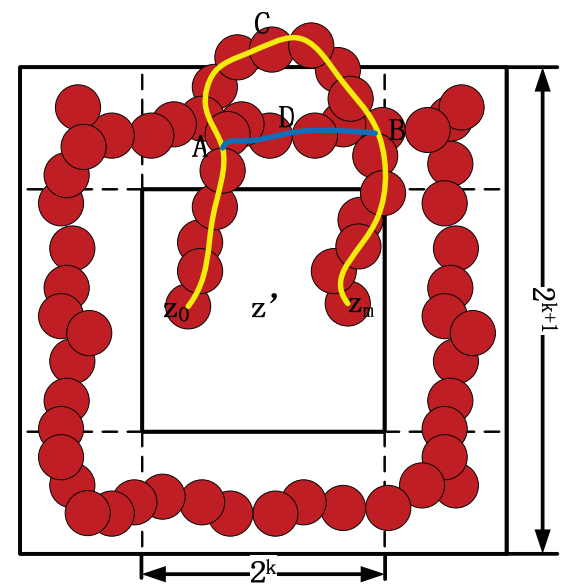

Fig. 5. The contradiction if the shortest path from $z_{0}$ to $z_{m}$ is not contained in $\left[z_{x}^{\prime}-2^{k}, z_{x}^{\prime}+2^{k}\right] \times\left[z_{y}^{\prime}-2^{k}, z_{y}^{\prime}+2^{k}\right]$.

Suppose $u, v, w$ are three consecutive nodes along this shortest path. Then $\|u-w\|>(1+\epsilon) r_{0}$, or we can eliminate node $v$, and get a shorter path. This also indicates that if we draw disks with radius $\frac{(1+\epsilon) r_{0}}{2}$ centering at $u$ and $w$ respectively, the two disks are disjoint. Assume the number of hops of the shortest path is $N$, then we can draw $\frac{L}{2}$ disjoint disks in total. And these disks are all located in a square with side length $2^{k+1}+(1+\epsilon) r_{0}$. Thus,

$$
\frac{L}{2} \cdot \pi\left(\frac{(1+\epsilon) r_{0}}{2}\right)^{2} \leq\left(2^{k+1}+(1+\epsilon) r_{0}\right)^{2} .
$$

Then,

$$
L \leq \frac{8\left(2^{k+1}+(1+\epsilon) r_{0}\right)^{2}}{\pi\left((1+\epsilon) r_{0}\right)^{2}}
$$

Note that $N_{\lambda_{L}}^{\prime}(0, m)$ is the minimum number of hops from $x_{0}$ to $x_{m}$, thus

$$
N_{\lambda_{L}}^{\prime}(0, m) \leq L \leq \frac{8\left(2^{k+1}+(1+\epsilon) r_{0}\right)^{2}}{\pi\left((1+\epsilon) r_{0}\right)^{2}}
$$

Now, if $N_{\lambda_{L}}^{\prime}(0, m)>\frac{8\left(2^{k+1}+(1+\epsilon) r_{0}\right)^{2}}{\pi\left((1+\epsilon) r_{0}\right)^{2}}$, then none of $C\left(2^{k_{0}+1}\right), C\left(2^{k_{0}+2}\right), \ldots, C\left(2^{k}\right)$ is good. Thus,

$$
\begin{aligned}
& \mathbb{P}\left(N_{\lambda_{L}}^{\prime}(0, m)>\frac{8\left(2^{k+1}+(1+\epsilon) r_{0}\right)^{2}}{\pi\left((1+\epsilon) r_{0}\right)^{2}}\right) \\
\leq & \prod_{i=k_{0}+1}^{k} \mathbb{P}\left(A_{C}^{c}\left(2^{i}\right)\right) \leq(1-\rho)^{k-k_{0}} .
\end{aligned}
$$

Let $l_{k}=\frac{8\left(2^{k+1}+(1+\epsilon) r_{0}\right)^{2}}{\pi\left((1+\epsilon) r_{0}\right)^{2}}$, then, 


$$
\begin{aligned}
& E\left(N_{\lambda_{L}}^{\prime}(0, m)\right) \\
= & \sum_{i=1}^{\infty} \mathbb{P}\left(N_{\lambda_{L}}^{\prime}(0, m) \geq i\right) \\
= & \sum_{i=1}^{l_{k_{0}}} \mathbb{P}\left(N_{\lambda_{L}}^{\prime}(0, m) \geq i\right)+\sum_{j=k_{0}}^{\infty} \sum_{i=l_{j}+1}^{l_{j+1}} \mathbb{P}\left(N_{\lambda_{L}}^{\prime}(0, m) \geq i\right) \\
\leq & l_{k_{0}}+\sum_{j=k_{0}}^{\infty} \mathbb{P}\left(N_{\lambda_{L}}^{\prime}(0, m)>l_{j}\right) \cdot\left(l_{j+1}-l_{j}\right) \\
< & l_{k_{0}}+\sum_{j=k_{0}}^{\infty}(1-\rho)^{j-k_{0}} \cdot \frac{8\left(2^{j+2}+(1+\epsilon) r_{0}\right)^{2}}{\pi\left((1+\epsilon) r_{0}\right)^{2}} \\
< & \infty
\end{aligned}
$$

\section{Proof of Lemma 9}

Proof: In previous analysis, we have proved that $\mathbb{P}\left(A_{C}\left(2^{k}\right)\right) \geq \rho$ whenever $k \geq k_{0}$. Summing over $k$ yields

$$
\sum_{k=k_{0}}^{\infty} \mathbb{P}\left(A_{C}\left(2^{k}\right)\right)=\sum_{k=k_{0}}^{\infty} \rho=\infty \text {. }
$$

Since $A_{C}\left(2^{k}\right), k=k_{0}, k_{0}+1, \ldots$ are independent events, according to the Borel-Cantelli Theorem, with probability 1, there exist $k^{\prime}<\infty^{9}$, such that $A_{C}\left(2^{k^{\prime}}\right)$ occurs.

We now construct squares $B_{1}$ and $B_{2}$ centered at $\frac{x_{m k}+x_{(m+1) k}}{2 \prime \prime}$ and $\frac{x_{(m+n) k}+x_{(m+n+1) k}}{2}$ with side length $2^{k^{\prime}+1}$ and $2^{k}+1$ respectively, such that the path with minimum number of hops from $x_{m k}$ to $x_{(m+1) k}$, and the path with minimum number of hops from $x_{(m+n) k}$ to $x_{(m+n+1) k}$ are contained in $B_{1}$ and $B_{2}$.

Due to the stationarity, $k^{\prime \prime}$ does not rely on $n$. Besides, $k^{\prime}$ and $k^{\prime \prime}$ are all finite(we denote this event by $A_{f}$ ) with probability 1 . Thus, when $n$ is large enough, $B_{1}$ and $B_{2}$ are disjointed. Hence, $N_{\lambda_{L}}^{\prime}(m k,(m+1) k)$ and $N_{\lambda_{L}}^{\prime}((m+n) k,(m+n+1) k)$ become independent.

Therefore,

$$
\begin{aligned}
& \lim _{n \rightarrow \infty} \mathbb{P}\left(\{ N _ { \lambda _ { L } } ^ { \prime } ( m k , ( m + 1 ) k ) < i \} \bigcap \left\{N_{\lambda_{L}}^{\prime}((m\right.\right. \\
+ & n) k,(m+n+1) k)<i\}) \\
= & \lim _{n \rightarrow \infty} \mathbb{P}\left(\{ N _ { \lambda _ { L } } ^ { \prime } ( m k , ( m + 1 ) k ) < i \} \bigcap \left\{N_{\lambda_{L}}^{\prime}((m\right.\right. \\
+ & \left.n) k,(m+n+1) k)<i\} \mid A_{f}\right) \mathbb{P}\left(A_{f}\right) \\
+ & \lim _{n \rightarrow \infty} \mathbb{P}\left(\{ N _ { \lambda _ { L } } ^ { \prime } ( m k , ( m + 1 ) k ) < i \} \bigcap \left\{N_{\lambda_{L}}^{\prime}((m\right.\right. \\
+ & \left.n) k,(m+n+1) k)<i\} \mid A_{f}^{c}\right) \mathbb{P}\left(A_{f}^{c}\right) \\
= & \mathbb{P}\left(\left\{N_{\lambda_{L}}^{\prime}(m k,(m+1) k)<i\right\} \mid A_{f}\right) \mathbb{P}\left(\left\{N_{\lambda_{L}}^{\prime}((m\right.\right. \\
+ & \left.n) k,(m+n+1) k)<i\} \mid A_{f}\right) \\
= & \mathbb{P}\left(\left\{N_{\lambda_{L}}^{\prime}(m k,(m+1) k)<i\right\}\right) \mathbb{P}\left(\left\{N_{\lambda_{L}}^{\prime}((m+\right.\right. \\
& n) k,(m+n+1) k)<i\})
\end{aligned}
$$

Then, $N_{\lambda_{L}}^{\prime}(m k,(m+1) k)$ is strong mixing.

${ }^{9}$ Actually, the number of such $k^{\prime}$ is infinite.

\section{Proof of Lemma 10}

Proof: Similar to that in the proof of lemma 8, we construct a series of squares centered at $(0, n)$ with side length $1,2,4, \ldots, 2^{k}, \ldots$ (Fig. 3). $C(d)$ is defined similarly. We say $C(d)$ is perfect if and only if $C(d)$ is good and the circuit in $C(d)$ belongs to the giant component.

Again, we apply Borel-Cantelli Theorem to Eqn. (24). With probability 1 , there exists $\left\{k^{\prime}\right\}$, such that event $A_{C}\left(2^{k^{\prime}}\right)$ occurs. Then, $d\left(z_{n},(0, n)\right)<2^{k^{\prime}}<\infty$ with probability 1 .

\section{E. Proof of Lemma 5}

Proof: According to Birkhoff Ergodic Theorem, we only need to show that $\left\{S_{g, t_{k}, u^{(k)}}(\lambda)\right\}_{k}$ is ergodic. In fact, we will show that $\left\{S_{g, t_{k}, u^{(k)}}(\lambda)\right\}_{k}$ is strong mixing, and thus ergodic.

Consider $S_{g, t_{k}, u^{(k)}}(\lambda)$ and $S_{g, t_{k+n}, u^{(k+n)}}(\lambda)$. We only need to show that for any constants $s_{1}, s_{2}>0$,

$$
\begin{aligned}
& \lim _{n \rightarrow \infty} \mathbb{P}\left(\left\{S_{g, t_{k}, u^{(k)}}(\lambda)<s_{1}\right\} \bigcap\left\{S_{g, t_{k+n}, u^{(k+n)}}(\lambda)<s_{2}\right\}\right) \\
& =\mathbb{P}\left(S_{g, t_{k}, u^{(k)}}(\lambda)<s_{1}\right) \mathbb{P}\left(S_{g, t_{k+n}, u^{(k+n)}}(\lambda)<s_{2}\right) .
\end{aligned}
$$

Note that all $S_{g, t_{k}, u^{(k)}}(\lambda)$ 's are equal in distribution to a random variable $S_{g}(\lambda)$, and $E\left(S_{g}(\lambda)\right)<\infty$ due to the subcriticality of RCM $\mathcal{G}_{t}\left(\lambda, r_{0}, g(r)\right)$. Therefore, for any fixed $\epsilon>0$, there exists $M_{\epsilon}$, such that

$$
\mathbb{P}\left(S_{g}(\lambda)>M_{\epsilon}\right)<\epsilon
$$

. We define $\hat{S}_{g, t_{k}, u^{(k)}}(\lambda)=\min \left\{S_{g, t_{k}, u^{(k)}}(\lambda), M_{\epsilon}\right\}$ for $k=$ $1,2, \ldots$ Then, $\hat{S}_{g, t_{k}, u^{(k)}}(\lambda)$ only depends on the realization of nodes and links in the circler region $H\left(u^{(k)}, M_{\epsilon}\right)$.

Consider the distance $d\left(u^{(k)}, u^{(k+n)}\right)$ between node $u^{(k)}$ and $u^{(k+n)}$. By the construction of $u^{(k)}$ 's, we know that as $n$ increases to infinity, $d\left(u^{(k)}, u^{(k+n)}\right)$ also increases to infinity. We choose $N>0$, such that for all $n>N, d\left(u^{(k)}, u^{(k+n)}\right)>$ $2 M_{\epsilon}$. Then $H\left(u^{(k)}, M_{\epsilon}\right)$ and $H\left(u^{(k+n)}, M_{\epsilon}\right)$ will become disjoint. By the spacial independence of Poisson Point Process, we then obtain that for any $n>N$,

$$
\begin{aligned}
& \mathbb{P}\left(\left\{\hat{S}_{g, t_{k}, u^{(k)}}(\lambda)<s_{1}\right\} \bigcap\left\{\hat{S}_{g, t_{k+n}, u^{(k+n)}}(\lambda)<s_{2}\right\}\right) \\
& =\mathbb{P}\left(\hat{S}_{g, t_{k}, u^{(k)}}(\lambda)<s_{1}\right) \mathbb{P}\left(\hat{S}_{g, t_{k+n}, u^{(k+n)}}(\lambda)<s_{2}\right) .(26)
\end{aligned}
$$

From the construction of $\hat{S}_{g, t_{k}, u^{(k)}}(\lambda)$, we know that for $s \leq M_{\epsilon}$,

$$
\mathbb{P}\left(S_{g, t_{k}, u^{(k)}}(\lambda)<s\right)=\mathbb{P}\left(\hat{S}_{g, t_{k}, u^{(k)}}(\lambda)<s\right) ;
$$

while for $s>M_{\epsilon}$,

$$
\mathbb{P}\left(M_{\epsilon} \leq S_{g, t_{k}, u^{(k)}}(\lambda)<s\right) \leq \epsilon
$$

Then, using Eqn. (26), it is easy to show that

$$
\begin{aligned}
& \lim _{n \rightarrow \infty} \mid \mathbb{P}\left(\left\{S_{g, t_{k}, u^{(k)}}(\lambda)<s_{1}\right\} \bigcap\left\{S_{g, t_{k+n}, u^{(k+n)}}(\lambda)<s_{2}\right\}\right) \\
& -\mathbb{P}\left(S_{g, t_{k}, u^{(k)}}(\lambda)<s_{1}\right) \mathbb{P}\left(S_{g, t_{k+n}, u^{(k+n)}}(\lambda)<s_{2}\right) \mid<2 \epsilon .
\end{aligned}
$$

Let $\epsilon \rightarrow 0$, we then obtain Eqn. (25). 


\section{DISCUSSION}

In this section, we make simulations to uphold our theoretical results. First, we give a further discussion on some parameters in our expressions. Then, enormous simulations are done to justify several assertions in this paper. Our theoretical results are based on a relatively general model, Random Connection Model. Many Network Models can be converted to a Random Connection Model, making our results applicable to many different cases. The difference is that the connection functions are different in different cases. In the following discussion, we simply let $r_{0}=1$, and the connection function $g$ be defined as

$$
g(r)=\left\{\begin{aligned}
\frac{1}{4}(2-r)^{2} & : \quad r \leq 1 \\
0 & : \quad r>1
\end{aligned}\right.
$$

Moreover, if we take propagation delay into consideration, we let $\tau=0.2$.

\section{A. Discussion on Several Parameters}

In our expression of theoretical bounds Eqn. (7), two terms $\kappa_{\epsilon}$ and $E\left(S_{g}(\lambda)\right)$ are applied. Besides, In Eqn. (8), $E\left(\min \left\{S_{g}(\lambda), \frac{r_{0}}{\tau}\right\}\right)$ is applied. $\kappa_{\epsilon}$ is a constant, and we simply obtain its value through simulation; while $E\left(S_{g}(\lambda)\right)$ and $E\left(\min \left\{S_{g}(\lambda), \frac{r_{0}}{\tau}\right\}\right)$ are functions depending on $\lambda$, and we find two analytical expressions to approximate them.

We first focus on $\kappa_{\epsilon}$ (defined in lemma 3 ). Here, we set $\epsilon=0.02$. In our simulation, we simulate 2304 points in a $40 \times 40$ region. The node density is $\lambda=\lambda_{L} \approx 1.44$, and the transmission range is $r_{0} \sqrt{1+\epsilon} \approx 1$.01. A packet is originated from a node located at the center of the region, we record down the minimum number of hops and the distance from the source for each node, and plot it in Fig. 6.(a).
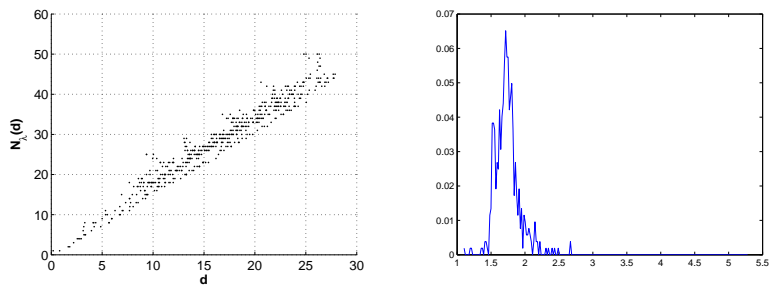

(a) Illustration of the relationship (b) The probability distribution between $N_{\lambda}(d)$ and $d$ graph of $\frac{N_{\lambda}(d)}{d}$

Fig. 6. Simulation results on $\kappa$. The first Figure reveals the linear relationship between $N_{\lambda}(d)$ and $d$, and the second indicates $\kappa_{\epsilon} \approx 1.7153$.

From Fig. 6.(a), we can see that $N_{\lambda}(d)$ grows linearly with $d$. To find $\kappa$, we calculate $\frac{N_{\lambda}(d)}{d}$ for each node, and present its probability distribution graph in Fig. 6.(b). It can be seen from Fig. 6.(b) that the probability $\frac{N_{\lambda}(d)}{d}=1.7153$ is the largest. Thus, $\kappa_{\epsilon} \approx 1.7153$.

Next, we turn to $E\left(S_{g}(\lambda)\right)$. The physical meaning of $E\left(S_{g}(\lambda)\right)$ is the average size of the connected component intersected with the origin. It is obvious that when $\lambda=0$, $E\left(S_{g}(\lambda)\right)=0$. And when $\lambda=\lambda_{I}, E\left(S_{g}(\lambda)\right)=\infty$ since the network is percolated in this case. Thus, we give a conjecture about the analytical expression of $E\left(S_{g}(\lambda)\right)$, i.e.,

$$
E\left(S_{g}(\lambda)\right)=\frac{c_{1} \lambda}{\lambda_{I}-\lambda} .
$$

We make enormous numerical computations to find the experimental values of $E\left(S_{g}(\lambda)\right)$ with respect to different $\lambda$, ranging from $1.5\left(\lambda_{L}(1+\epsilon)<1.5\right)$ to 2.4 . We then rewrite Eqn. (27) as

$$
\frac{1}{E\left(S_{g}(\lambda)\right)}=\frac{\lambda_{I}}{c_{1}} \cdot \frac{1}{\lambda}-\frac{1}{c_{1}} .
$$

Using the least square fitting, we can easily obtain $c_{1} \approx$ $1.2841, \lambda_{I} \approx 2.4886$.

Then we make a comparison between the fitting value and the experimental value of $E\left(S_{g}(\lambda)\right)$. From Fig. 7, It can be seen that there is a good agreement between fitting and experimental results.

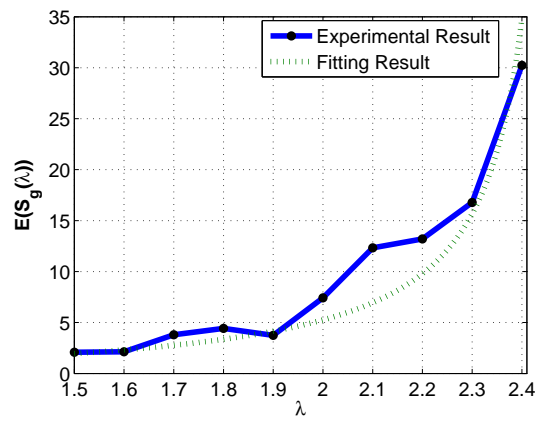

Fig. 7. Comparison between experimental and fitting value of $E\left(S_{g}(\lambda)\right)$.

Now, we come to $E\left(\min \left\{S_{g}(\lambda), \frac{r_{0}}{\tau}\right\}\right)$. Obviously, $E\left(\min \left\{S_{g}(\lambda), \frac{r_{0}}{\tau}\right\}\right)=0$ whenever $\lambda=0$. Besides, $E\left(\min \left\{S_{g}(\lambda), \frac{r_{0}}{\tau}\right\}\right) \leq \frac{r_{0}}{\tau}, \forall \lambda$, and $E\left(\min \left\{S_{g}(\lambda), \frac{r_{0}}{\tau}\right\}\right)$ is monotone increasing with $\lambda$. Thus, we conjecture that the analytical expression of $E\left(\min \left\{S_{g}(\lambda), \frac{r_{0}}{\tau}\right\}\right)$ has the format

$$
E\left(\min \left\{S_{g}(\lambda), \frac{r_{0}}{\tau}\right\}\right)=\frac{c_{2} \lambda}{c_{3}+\lambda} .
$$

Similarly, we conduct numerical computations to find the experimental values of $E\left(\min \left\{S_{g}(\lambda), \frac{r_{0}}{\tau}\right\}\right)$ with respect to different $\lambda$, ranging from 1.44 to 20. We rewrite Eqn. (28) as

$$
\frac{1}{E\left(\min \left\{S_{g}(\lambda), \frac{r_{0}}{\tau}\right\}\right)}=\frac{c_{3}}{c_{2}} \cdot \frac{1}{\lambda}+\frac{1}{c_{2}} .
$$

Using the least square fitting, we can easily obtain $c_{2} \approx$ $2.0845, c_{3} \approx 1.6813$.

Then we make a comparison between the fitting value and the experimental value of $E\left(\min \left\{S_{g}(\lambda), \frac{r_{0}}{\tau}\right\}\right)$. From Fig. 8, It can be seen that there is a good agreement between fitting and experimental results.

\section{B. Comparison between Two Bounds}

This paper is originated from the idea that the delay-distance ratio $\gamma(\lambda)$ may depend on the node density $\lambda$. We calculate $\gamma(\lambda)$ under different node densities. From Fig. 9, we can see that $\gamma(1.6)=\lim _{d \rightarrow \infty} \frac{T_{\lambda}(d)}{d} \approx 0.68, \gamma(1.9) \approx 0.35, \gamma(2.2) \approx$ 


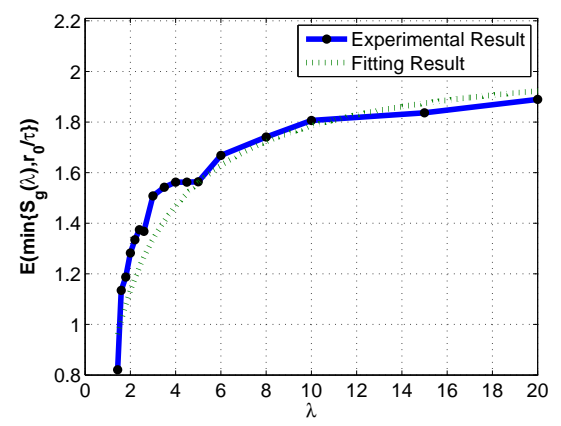

Fig. 8. Comparison between experimental and fitting value of $E\left(\min \left\{S_{g}(\lambda), \frac{r_{0}}{\tau}\right\}\right)$.

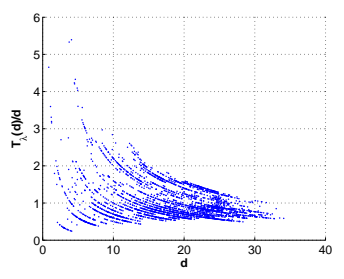

(a) $\lambda=1.6$

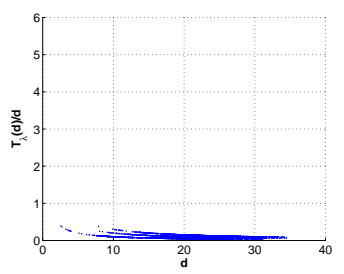

(c) $\lambda=2.2$

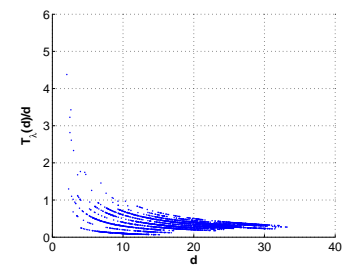

(b) $\lambda=1.9$

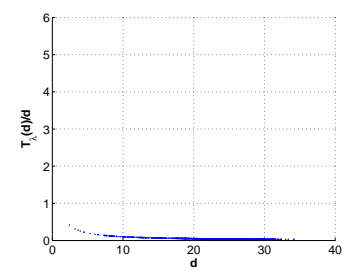

(d) $\lambda=2.5$
Fig. 9. Simulation results on different $\lambda$.

$0.11, \gamma(2.5) \approx 0$. This justifies the fact that $\gamma(\lambda)$ is monotone decreasing with respect to $\lambda$.

Now, we are ready to compare our theoretical bounds and the experimental values of $\gamma(\lambda)$. We first ignore the propagation delay, Fig. 10 shows the comparison between the experimental value and our theoretical value of both the upper bound and the lower bound. In our simulations, We work out the experimental values of $\gamma(\lambda)$ where $\lambda$ is set to be evenly distributed in the interval of $\left[\lambda_{L}(1+\epsilon), \lambda_{I}\right]$. From Fig. 10, we find that the experimental values are right bounded by both the upper and the lower bounds.

Then, we take propagation delay into consideration. In the following part, we take $\tau=0.2$. We first examine the effect of introducing propagation delay to the delay-distance ratio $\gamma(\lambda)$.

We consider two networks, whose node densities $\lambda$ are 1.5 and $2.2\left(1.5\right.$ is near $\lambda_{L}, 2.2$ is near $\left.\lambda_{I}\right)$ respectively. For each network, we make simulations to find $\gamma(\lambda)$ in the cases $\tau=0$ and $\tau=0.2$. The result is show in Fig. 11 .

From Fig. 11, we can also see that when $\lambda$ is small, the influence of $\tau$ to $\gamma(\lambda)$ is small; and the influence becomes more significant as $\lambda$ grows larger. This also indicate that when the node density is small, delay is mainly caused by

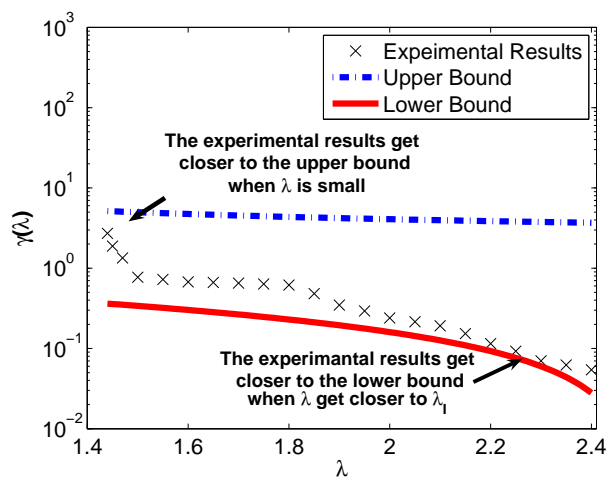

Fig. 10. Comparison between upper bound and lower bound(propagation delay is ignored).
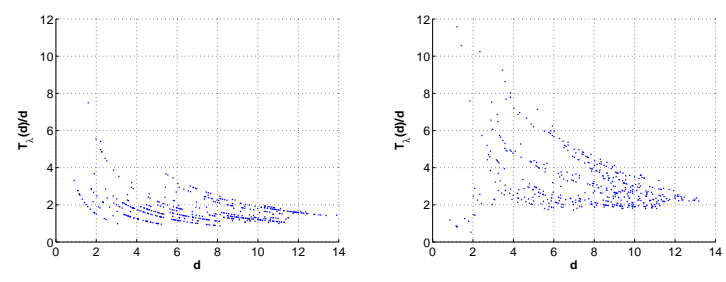

(a) $\lambda=1.5, \tau=0, \gamma(\lambda) \approx$ (b) $\lambda=1.5, \tau=0.2, \gamma(\lambda) \approx$ 1.8

2.1
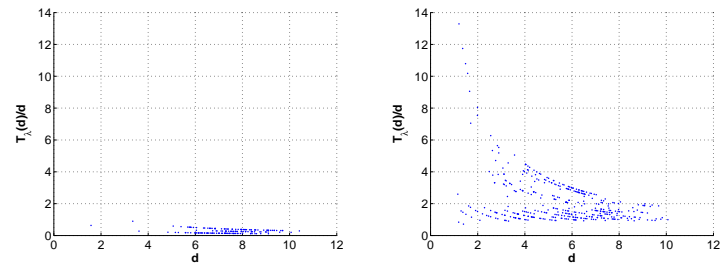

(c) $\lambda=2.2, \tau=0, \gamma(\lambda) \approx$ (d) $\lambda=2.2, \tau=0.2, \gamma(\lambda) \approx$ 0.1

1.8

Fig. 11. The influence of propagation delay $\tau$ to the delay-distance ratio $\gamma(\lambda)$.

the waiting delay (waiting delay is mainly caused by the loss of wireless connections); while when the node density is large, delay is mainly caused by the propagation delay.

Next, we compare our theoretical bounds and experimental results of $\gamma(\lambda)$ in the case $\tau=0.2$. In our simulation, the node densities $\lambda$ are chosen from [1.5,20]. Since the change of $\gamma(\lambda)$ is larger when $\lambda$ is small, we choose more simulation points for smaller node density. The comparison between our theoretical bounds and experimental results is shown in Fig. 12. The experimental values are right bounded by both the upper and the lower bounds.

From Fig. 10 and Fig. 12, it can be seen that when $\lambda$ is large, $\gamma(\lambda)$ is much closer to the lower bound. An explanation to this phenomena is that the larger the node density $\lambda$ is, the larger the size of clusters in the cluster to cluster transmission process. Larger cluster size provides more opportunity for forwarding packets. Thus, when the node density is large enough, it is probably that the message can transmit again right at the next time slot. This makes our lower bound more accurate. However, our upper bound is a little bit loose. But 


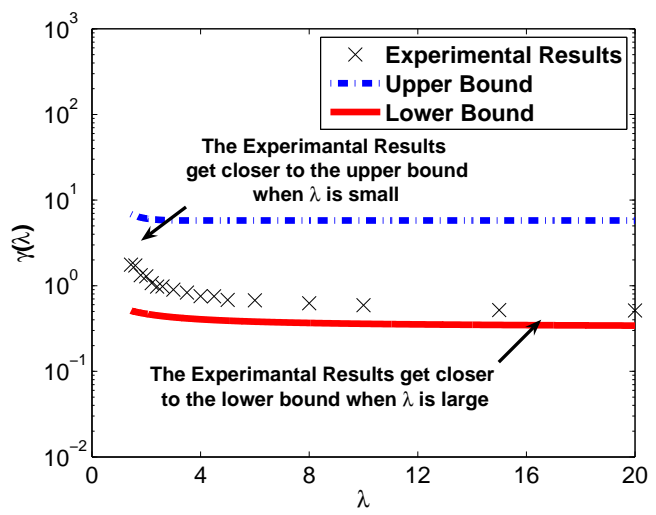

Fig. 12. Comparison between upper bound and lower bound(propagation delay is considered).

we can see that $\gamma(\lambda)$ gets closer to the upper bound when the node density $\lambda$ decreases. This is because our upper bound is obtained from the delay of just one path. And the smaller $\lambda$ is, the smaller the number of paths connecting two nodes. This makes $\gamma(\lambda)$ get closer to the upper bound.

\section{CONCLUSION}

In this paper, we study the fundamental relationship between $\gamma(\lambda)$ and $\lambda$ using percolation theory. We point out that unreliability of wireless connections brings about waiting delay and analytically characterize the upper and lower bound for $\gamma(\lambda)$. Then we take propagation delay into consideration, and obtain further results. Finally, through simulations based on the exact value of $\gamma(\lambda)$, we further obtain a new observation that the lower bound serves as a good approximation to the value of $\gamma(\lambda)$ in dense networks. And $\gamma(\lambda)$ gets closer to the upper bound when $\lambda$ decreases. Simulation results conform our theoretical findings.

\section{ACKNOWLEDGMENT}

This paper is supported by National Fundamental Research Grant (No. 2011CB302701); NSF China (No. 61271219); Shanghai Basic Research Key Project (No. 11JC1405100); China Ministry of Education Fok Ying Tung Fund (No. 122002); China Ministry of Education New Century Excellent Talent (No. NCET-10-0580).

\section{REFERENCES}

[1] "Next-Generation Wireless Mesh Networks: Combining a multi-radio architecture with high-performance routing to optimize video surveillance and other multimedia-grade applications," in Aruba Networks, Mar. 2011. [online]. Available: http://www.arubanetworks.com/pdf/technolo gy/whitepapers/wp_wirelessmesh.pdf

[2] J.M.Hammersley and D.J.A.Welsh, "First Passage Percolatin, Subadditive Processes, Stacastic Networks, and Generalized Renewal Theory," in Proc. Internat. Res. Semin., Statist. Lab., Univ. California, Berkeley, Calif., pages 61-110. Springer-Verlag, New York, 1965.

[3] Z. Kong and E. M. Yeh, "On the Latency for Information Dissemination in Mobile Wireless Networks," in Proc. ACM MobiHoc'08, Hong Kong SAR, China, May. 2008.

[4] P. Gupta and P. R. Kumar, "Critical Power for Asymptotic Connectivity in Wireless Networks," in Stochastic Analysis, Control, Optimization and Applications: A Volume in Honor of W.H. Fleming, W. M. McEneany, G. Yin, Q. Zhang, Eds. Boston, MA: Birkhauser, pp. 547-566, 1998.
[5] P. Wan and C. W. Yi, "Asymptotic Critical Transmission Radius and Critical Neighbor Number for K-connectivity in Wireless Ad Hoc Networks," in Proc. 5th ACM int. symp. Mobile Ad Hoc Networking and Computing, Roppongi Hills, Tokyo, Japan, May 24-26, 2004.

[6] M. D. Penrose, "On K-connectivity for a Geometric Random Graph," Random Structure and Algorithms, vol. 15, no. 2, pp. 145-164, Sept. 1999.

[7] Q. Wang, X. Wang and X. Lin, "Mobility Increases the Connectivity of K-hop Clustered Wireless Networks," ACM Mobicom'09, Beijing, Sept. 2009.

[8] D. Goeckel, B. Liu, D. Towsley, L. Wang and C. Westphal, "Asymptotic connectivity properties of cooperative wireless ad hoc network," $A C M$ Mobihoc'08, May. 2008.

[9] O. Dousse, F. Baccelli and P. Thiran, "Impact of Interferences on Connectivity in Ad Hoc Networks," in Proc. of IEEE Infocom, pp. 17241733, Apr. 2005.

[10] G. R. Grimmett, "Percolaiton,” Springer, 1999.

[11] O. Dousse, P. Mannersalo and P. Thiran, "Latency of Wireless Sensor Networks with Uncoordinated Power Saving Mechnisms," in Proc. ACM MobiHoc'04, pp. 129-120, May. 2004.

[12] Z. Kong, and E. M. Yeh. "Connectivity and Information Dissemination in Large-Scale Wireless Networks with Dynamic Links," submitted to IEEE Trans. Inform. Theory, 2008.

[13] D. J. Daley and D. Vere-Jones, "An Introduction to the Theory of Point Process," New York: Springer, 1988.

[14] J.F.C. Kingman, "Poisson Processes," Clarendon Press, Oxford, 1993.

[15] R. Meester and R. Roy, "Continuum Percolation," NewYork: Cambridge University Press, 1996.

[16] T. Liggett, "An Improved Subadditive Ergodic Theorem," Annals of Prob., vol. 13, pp. 1279-1285, 1985.

[17] W.Ren, Q.Zhao, and A.Swami, "On the Connectivity and Multihop Delay of Ad Hoc Cognitive Radio Networks" to appear in Proc. of IEEE International Conference on Communications(ICC), May, 2010.

[18] A. Baddeley, "Spatial point processes and their applications," Lecture Notes in Mathematics, 1892:1-75, 2007.

[19] S. Zhao, L. Fu, X. Wang, Q. Zhang, "Fundamental Relationship between Node Density and Delay in Wireless Ad Hoc Networks with Unreliable Links," ACM MobiCom 2011, Las Vegas, Sept 2011.

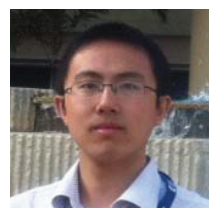

Shizhen Zhao received the BE degree in electronic engineering from Shanghai Jiao Tong University, China in 2010, and is pursuing a Ph.D. degree at the school of ECE, Purdue University, West Lafayette, IN, USA. His research interests focus on the scheduling problem in wireless networks and smart grid.

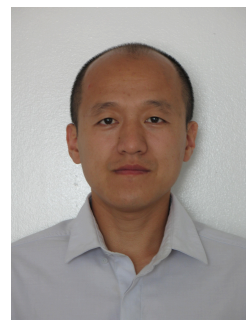

Xinbing Wang received the B.S. degree (with hons.) from the Department of Automation, Shanghai Jiaotong University, Shanghai, China, in 1998, and the M.S. degree from the Department of Computer Science and Technology, Tsinghua University, Beijing, China, in 2001. He received the Ph.D. degree, major in the Department of electrical and Computer Engineering, minor in the Department of Mathematics, North Carolina State University, Raleigh, in 2006. Currently, he is a professor in the Department of Electronic Engineering, Shanghai Jiaotong University, Shanghai, China. Dr. Wang has been an associate editor for IEEE/ACM Transactions on Networking and IEEE Transactions on Mobile Computing, and the member of the Technical Program Committees of several conferences including ACM MobiCom 2012, ACM MobiHoc 2012, 2013, IEEE INFOCOM 2009-2014. 